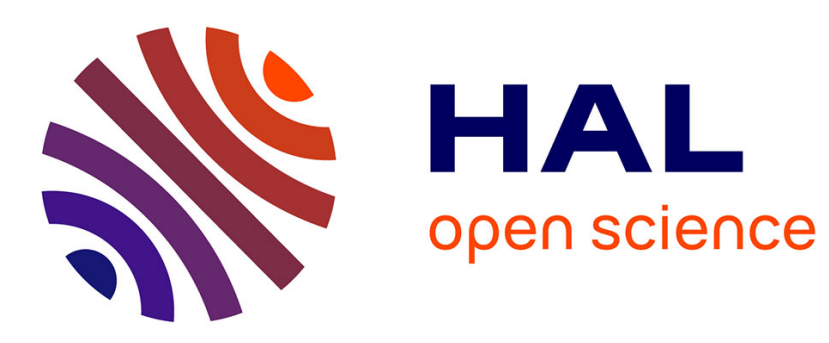

\title{
Les dispositifs judiciaires des Cyclades à l'époque classique et la question de leur indépendance
}

\author{
Alexandra Bartzoka
}

\section{To cite this version:}

Alexandra Bartzoka. Les dispositifs judiciaires des Cyclades à l'époque classique et la question de leur indépendance. KTÈMA Civilisations de l'Orient, de la Grèce et de Rome antiques, 2018, Luxe et richesse dans l'Antiquité et à Byzance, 43, pp.133-154. halshs-01959971

\section{HAL Id: halshs-01959971 https://shs.hal.science/halshs-01959971}

Submitted on 19 Dec 2018

HAL is a multi-disciplinary open access archive for the deposit and dissemination of scientific research documents, whether they are published or not. The documents may come from teaching and research institutions in France or abroad, or from public or private research centers.
L'archive ouverte pluridisciplinaire HAL, est destinée au dépôt et à la diffusion de documents scientifiques de niveau recherche, publiés ou non, émanant des établissements d'enseignement et de recherche français ou étrangers, des laboratoires publics ou privés. 

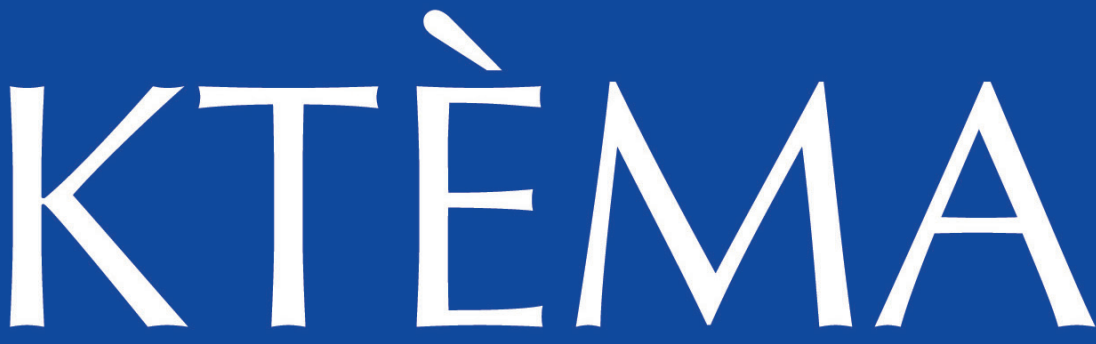

\section{CIVILISATIONS DE L'ORIENT, DE LA GRÈCE ET DE ROME ANTIQUES}

Philippe QuenET

Sylvie Donnat

Anne-Marie AdAM

Alain CHAuvot

Catherine Duvette

Stavros LAZARIS

Francesco MARI

Alexandra BARTZOKA

Edmond LÉvy

\section{Luxe et richesse dans l'Antiquité et à Byzance}

Luxe et transgression dans les cités-États sud-mésopotamiennes (3100-2350 av. J.-C.) d'après quelques séries d'objets en coquille .5

Du luxe aux richesses-špss. À propos de la scène du petit lever de Ptahhotep (Égypte, vers 2400-2300 av. J.-C.)

Luxe du cadre de vie et du cadre de mort chez les Celtes de l'âge du fer.......47

Luxus et pompa

La notion de luxe d'après trois portraits de «barbares»

dans la Correspondance de Sidoine Apollinaire

Une idée du luxe en contexte paysan:

le cas des villages protobyzantins du ğebel Zawiyé

(Massif calcaire de Syrie du Nord).

Sur le statut et l'utilisation de l'or à Byzance:

le cas des manuscrits chrysographiés

\section{Varia}

Les sens de la poignée de main en Grèce ancienne du VIII $^{\mathrm{e}}$ au v $\mathrm{v}^{\mathrm{e}}$ siècle avant J.-C.

Les dispositifs judiciaires des Cyclades à l'époque classique et la question de leur indépendance.

Bía chez Aristote 


\section{KTÈMA \\ CIVILISATIONS DE L'ORIENT, DE LA GRÈCE ET DE ROME ANTIQUES \\ Revue annuelle \\ Fondateurs : Edmond Frézouls † \\ Edmond LÉvy}

Directrice de la revue: Dominique LENFANT

Directeur honoraire: Edmond LÉvY

Comité de rédaction: Agnès Arbo Molinier, Frédéric Colin, Michel Humm, Anne Jacquemin, Luana Quattrocelli, Anne-Caroline Rendu-Loisel

Comité scientifique: Cinzia Bearzot (Milan), Harriet Flower (Princeton), Sabine Huebner (Bâle), Tanja Itgenshorst (Fribourg, Suisse), Olaf Kaper (Leyde), Alexander PRUß (Mayence), Christopher Tuplin (Liverpool), Ralf von DEN Hoff (Fribourg, Allemagne)

Comité de lecture: Le comité de lecture est constitué des spécialistes extérieurs qui expertisent les articles et doivent rester anonymes.

Directeur de publication: Michel Deneken, président de l’Université de Strasbourg

Maquette et mise en page: Ersie LERIA

\section{Éditeur}

Presses universitaires de Strasbourg

5 allée du Général Rouvillois - CS 50008

FR-67083 Strasbourg CEDEX

Tél. : (33) 0368856265

info.pus@unistra.fr

pus.unistra.fr

\section{Ventes au numéro}

En librairie ou en commande en ligne sur le site des Presses universitaires de Strasbourg:

pus.unistra.fr

\author{
Abonnements \\ FMSH Diffusion/CID \\ 18 rue Robert-Schuman \\ CS 90003 \\ FR-94227 Charenton-Le-Pont Cedex \\ Tél.: 0153485630 \\ Fax: 0153482095 \\ cid@msh-paris.fr
}




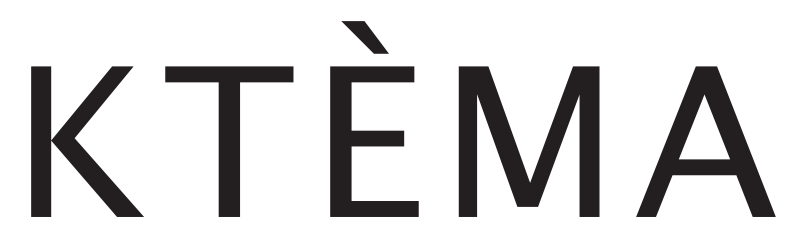

CIVILISATIONS DE L'ORIENT, DE LA GRÈCE ET DE ROME ANTIQUES

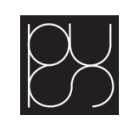

PRESSES UNIVERSITAIRES DE STRASBOURG

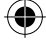




\section{Les dispositifs judiciaires des Cyclades à l'époque classique et la question de leur indépendance}

RÉsumé-. La présente étude aborde la question de l'existence ou non d'interactions dans le domaine de la justice entre Athènes et les Cyclades pendant l'époque classique. Les informations disponibles sont disparates et fragmentaires. Les termes utilisés pour désigner le dispositif judiciaire des îles qui appartiennent au complexe insulaire des Cyclades présentent des similitudes avec ceux d'Athènes, mais il n'est pas facile de savoir s'ils se réfèrent aux mêmes réalités procédurales que celles qui existaient à Athènes. Quant à leurs différences, elles montrent qu'Athènes n'a pas nécessairement fourni un modèle qui a été adopté par les régions voisines.

Aвstract-. This paper considers the interactions between Athenian judicial practices and those of the poleis of the Cycladic islands in the classical period. The evidence is very fragmentary. It often presents similarities in vocabulary, but it is difficult to determine in these cases whether similar words denote similar procedures. Regarding the differences among them, they show that Athens did not necessarily serve as a model that was adopted by neighbouring regions.

L'une des principales questions qui se posent quand on étudie l'évolution historique des cités grecques tient à l'évolution de leur système de justice, qui se construit progressivement afin que les normes et les lois, d'une part, réglementent les relations entre les différentes composantes de la cité et, d'autre part, répondent aux nécessités de chaque période. Du côté athénien, il est plus facile de retracer cette évolution, même s'il subsiste encore des lacunes importantes dans notre documentation. Au contraire, du côté des autres cités du monde grec, à quelques exceptions près, on est tributaire de sources peu nombreuses et fragmentaires et des comparaisons que l'on peut en faire avec les sources athéniennes. À cette difficulté s'ajoute la question de l'unité du droit grec (ou de la pluralité des droits grecs). Sur ce point, les avis des chercheurs sont variés: certains expliquent les éléments communs repérés dans les droits des cités comme des manifestations de l'existence de certains principes qualifiés de "grecs» ou les attribuent à la prédominance du droit attique, tandis que d'autres relèvent le caractère indépendant et spontané des points de convergence entre ces droits ${ }^{1}$. La présente étude s'inscrit dans cette problématique. Elle aborde la question de l'existence

Sauf indication contraire, les textes littéraires sont ceux proposés par la Collection des Universités de France (CUF). En ce qui concerne les traductions des textes littéraires et des inscriptions, j'indique à chaque fois le nom du traducteur. Lorsqu'aucun traducteur n'est mentionné, il s'agit de mes propres traductions.

(1) Par ex. Wolff 1972, p. 131-144, admet l'unité du droit grec et met en valeur l'importance des inscriptions pour examiner les similarités et les différences entre les droits des cités; FinLEY 1975, p. 136-146, au contraire, reste très sceptique vis-à-vis de l'existence de cette unité; RHODEs, LEwis 1997 proposent de voir des similarités dans les institutions politiques; 
ou non d'interactions dans le domaine de la justice entre Athènes et les cités des Cyclades à l'époque classique, afin de voir si l'on peut finalement arriver à des conclusions à propos des aspects judiciaires de ces îles ${ }^{2}$. À l'époque classique, c'est le Ive siècle qui fournit la plupart des exemples.

\section{LES PARTICULARITÉS DES CYCLADES}

Les Cyclades présentent un intérêt particulier pour notre étude pour trois raisons. La première est liée au fait que toutes les îles pour lesquelles on dispose d'informations appartiennent à la Ligue de Délos ${ }^{3}$ (478/7) et que la plupart d'entre elles sont membres de la seconde Confédération maritime $(377)^{4}$. Elles se situent ainsi dans un environnement de coexistence et d'imposition de l'archè athénienne, dans lequel les relations entre Athènes et ses alliés ont été renouvelées à la fois au niveau politique, économique et judiciaire. Par exemple, le décret qui formalise la seconde Confédération maritime (désigné dorénavant comme « décret d’Aristotélès») (377) ${ }^{5}$ constitue une preuve caractéristique des rapports qui ont été développés entre ses membres au niveau judiciaire et des questions qui en résultent.

Il stipule en effet que celui qui proposera ou mettra aux voix une proposition entrant en contradiction avec le décret et supprimant l'une de ses clauses sera jugé par «les Athéniens et les alliés» pour avoir brisé l'alliance, et que des peines lui seront imposées ${ }^{6}$. L'expression «les

Rubinstein 2003, p. 107-112, en examinant les attestations de l'institution de l'accusateur volontaire dans le monde grec, y voit un phénomène grec qui présente à la fois des similarités en ce qui concerne ses caractéristiques de base et des variations locales; GAGARIN 2005, p. 34-36, penche pour l'unité du droit grec en ce qui concerne les procédures judiciaires; HARRIs 2006, p. 3-4, parle de plusieurs caractéristiques communes dans les lois des cités grecques, qui, considérées ensemble, manifestent un nombre de principes partagés parmi ces communautés, en dépit de leurs différences; Low 2007, p. 83-102, s'interroge sur l'existence d'un droit grec international; MAGNetTo 2016, p. 34, discute l'institution des juges étrangers et montre ses implications pour l'unité du droit grec.

(2) Les Cyclades sont choisies comme ensemble d'étude pour les raisons qui sont évoquées ci-après.

(3) Sur l'histoire de la Ligue de Délos, voir Meiggs 1972 (même si les datations proposées pour certains décrets sont maintenant contestées, son œuvre reste un point de référence); Scheibelreiter 2013. Sur la politique athénienne de promouvoir ou de ne pas promouvoir le régime démocratique au sein de la Ligue, voir Brock 2009, p. 149-166. Pour un résumé des finances de la Ligue, voir Migeotte 2014, p. 438-443, avec des références aux problèmes soulevés et à la bibliographie antérieure. Sur les relations judiciaires, il faut distinguer: 1. Les symbolai, conventions conclues entre Athènes et les cités alliées, au terme desquelles était réglée la manière dont les procès devaient se dérouler en cas de conflit judiciaire. Leur jugement éventuel à Athènes n'a aucun rapport avec la politique du transfert des procès à Athènes. Sur cette distinction, voir Ste Croix 1961, p. 94-95. Sur ces conventions, voir aussi GaUthier 1972. 2. Le transfert devant les tribunaux athéniens des affaires qui concernaient les alliés (affaires des alliés qui étaient considérées par l'Assemblée athénienne comme suffisamment importantes pour être transférées à Athènes, affaires qui concernaient le tribut des alliés, ainsi que le respect des décrets athéniens par les cités alliées). Les inscriptions correspondantes présentent des difficultés d'interprétation et de datation, en raison de leur état fragmentaire. Pour un sommaire des datations proposées, voir RHODEs 2008, p. 500-506; PÉBARTHe 2011, p. 59-73. On cite, à titre indicatif, les inscriptions suivantes: $I G \mathrm{I}^{3}, 14,15,21,34,40,68,71$.

(4) Pour chacune de ces cités, voir les chapitres correspondants dans HANSEN - Nielsen 2004; BonNin 2015, surtout p. 81 (tableau), 247-248, 252-253 (discussion sur le rattachement des cités Ios, Naxos et Théra à la seconde Confédération maritime). Sur la nature des activités hégémoniques dans l’archipel cycladique, voir BonNIN - LE QUÉRÉ 2014.

(5) $\mathrm{R} \& \mathrm{O}, \mathrm{n}^{\circ} 22$.

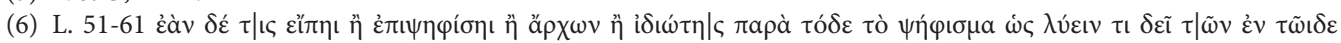

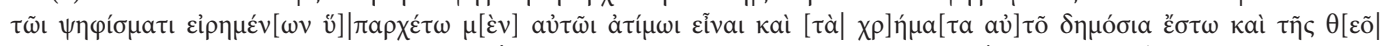

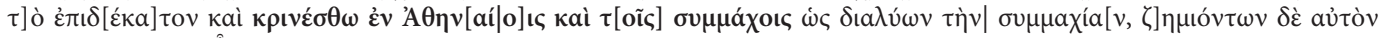

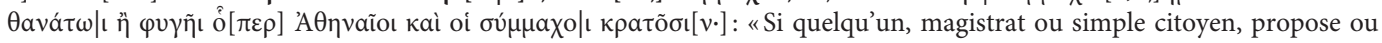
met aux voix, contrairement au présent décret, qu'on doit supprimer une des clauses inscrites sur le décret, qu'il soit frappé d'atimie et que ses biens soient confisqués et que la dîme soit consacrée à la déesse et qu'il soit jugé devant les Athéniens et les alliés pour avoir brisé l'alliance. Qu'il soit condamné à mort ou à l'exil partout où les Athéniens et les alliés sont souverains.» (trad. P. BRUN, légèrement modifiée). 
Athéniens et les alliés» ne précise pas le corps qui rendra la justice. Sur ce point, deux hypothèses peuvent être proposées: soit il s'agit d'un tribunal commun, composé d'Athéniens et d'alliés ${ }^{7}$, soit il s'agit d'un jugement rendu séparément par les Athéniens et par les alliés ${ }^{8}$. Quelle que soit l'option qu'on choisira, l'expression met l'accent sur le fait que l'ensemble des alliés d'une part, les Athéniens et les alliés de l'autre, peuvent constituer dans certaines circonstances une communauté juridique. En outre, quand on aborde l'interdiction faite aux Athéniens d'acheter, d'acquérir ou de prendre en gage une propriété sur le territoire des alliés, on utilise le terme $\varphi \tilde{n} v a ı$ pour désigner la procédure qui sera suivie au cas où cette clause ne serait pas respectée, et l'on propose que la dénonciation soit déposée devant les synedroi des alliés9. Les synedroi s'occuperont de la remise du bien: le dénonciateur aura la moitié de la somme acquise par la vente du bien et l'autre moitié sera propriété commune des alliés. Dans ce cas, la compétence judiciaire appartient aux alliés ${ }^{10}$ et, notamment, aux synedroi des alliés, qui sont les représentants de chaque allié et qui composent le synedrion, dans lequel toutes les cités, sauf Athènes, semble-t-il, ont un droit de vote ${ }^{11}$. La présence du terme $\varphi \tilde{\eta} v a ı$ dans un décret qui se devait d'être accessible à toute la communauté montre que c'est un lexique partagé par l'ensemble de la Confédération, sans indiquer clairement l'origine de cette extension. Faut-il y voir une importation en provenance d'Athènes qui marquerait la prédominance du droit attique, ou le signe d'une similarité entre les systèmes juridiques de différentes communautés? À l'appui de la première hypothèse, les sources attiques attestent l'existence de la procédure de phasis, qui était très courante dans les tribunaux athéniens depuis le $\mathrm{V}^{\mathrm{e}}$ siècle, pour diverses infractions: infractions relatives au commerce et à la propriété publique, violation des lois qui protègent les orphelins, affaires d'impiété ${ }^{12}$. Pour un certain nombre de ces affaires (surtout celles qui concernent des biens), on sait que si l'accusé était reconnu coupable, le bien ou la propriété en litige était confisqué et vendu et que le dénonciateur recevait la moitié de la somme acquise par la vente du bien ${ }^{13}$, et la clause judiciaire citée dans le décret d'Aristotélès fait partie de cette catégorie. La seconde hypothèse, celle de l'existence d'une procédure partagée par différentes communautés, pourrait quant à elle être étayée par les occurrences du terme renvoyant à la phasis signalées à la même période dans d'autres régions du monde grec qui n'appartiennent pas à la sphère athénienne ${ }^{14}$.

La deuxième raison qui conduit à distinguer pour cette étude les Cyclades des autres alliés d'Athènes tient à leur géographie. Ces îles ne sont pas seulement à proximité d'Athènes, mais aussi proches les unes des autres ${ }^{15}$. Cette particularité peut créer des relations d’interdépendance entre

(7) Voir Accame 1941, p. 138-140 (un tribunal composé d'alliés et d'un nombre d'Athéniens qui serait peut-être le même que les alliés); CARLIER 1995, p. 47.

(8) Voir Larsen 1968, p. 63-64; Cargill 1981, p. 121-122; Dreher 1989, p. 278; Lewis 1997, p. 148 n. 39 ; R \& O, p. $102, \mathrm{n}^{\circ} 22$.

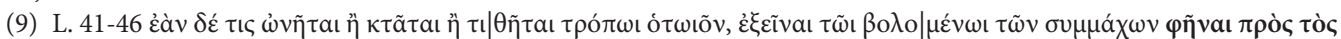

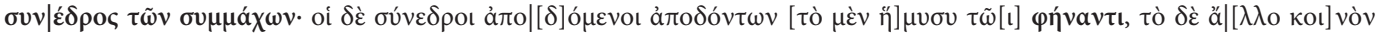

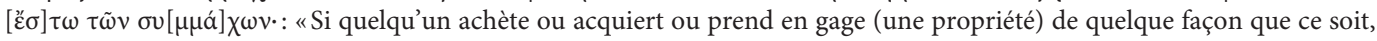
qu'il soit permis à celui qui le veut parmi les alliés de le dénoncer devant les synedroi des alliés. Que les synedroi, après avoir vendu (le bien), remettent la moitié au dénonciateur et que l'autre moitié soit propriété commune des alliés.». Cf. LARSEN 1968, p. 62-63.

(10) Cf. Cargill 1981, p. 123.

(11) Diodore de Sicile, XV. 28. 3-4. Cf. Cargill 1981, p. 116 et n. 2.

(12) Voir, à titre indicatif, SEG 30.61, 1. 28; ArIStophane, Acharniens, v. 819-827, 908-913, Cavaliers, v. 300-302; Démosthène, Contre Lacritos, 51, Contre Théocrinès, 5, 7; Hypéride, Pour Euxénippos, 35; Isocrate, Contre Callimachos, 6; Lycurgue, Contre Léocrate, 27.

(13) Voir MacDowell 1991, p. 187-198. L’article de MacDowell est aussi discuté par Wallace 2003, p. 167-181.

(14) Voir en Arcadie: Mantinée: IPArk 9, 1. 22, 23, 24-25 (IV siècle), Tégée: IPArk 3, 1. 24 (IV siècle).

(15) Sur les conditions géographiques en tant que facteurs déterminant les interactions entre les îles, voir Constantakopoulou 2007, p. 25-28; ead. 2017, p. 22-25. 
elles, relations qui, comme le montrent les sources disponibles, concernent certains aspects de leur dispositif juridique. Mis à part les dispositions de leur régime dotal et successoral, que nous discuterons ci-après, on sait par Isocrate, par exemple, que la loi qui réglait le statut de citoyen à Siphnos était inspirée d'un archétype emprunté à Kéos ${ }^{16}$.

La dernière raison repose sur le nombre et le caractère de sources disponibles qui concernent ces îles. D'une part, des décrets non attiques témoignent de l'existence de procédures judiciaires et de magistrats à compétence judiciaire qui portent les mêmes noms que ceux d'Athènes. D'autre part, la recherche archéologique a mis au jour du matériel à fonction judiciaire qui présente des similitudes avec celui d'Athènes. Par conséquent, se pose la question de savoir si les termes qui sont utilisés pour désigner le système de justice dans un cadre non athénien ainsi que le matériel découvert se réfèrent aux mêmes réalités procédurales que celles qui existent à Athènes ${ }^{17}$. Si l'on regarde, par exemple, les témoignages qui concernent le dispositif juridique, et pas seulement judiciaire, des Cyclades, on voit bien que ceux-ci entrent aussi dans la discussion autour de la prédominance ou non du droit attique et que nous sommes confrontés au même type de difficultés. Pour n'évoquer qu'une de ces difficultés, on lit dans un passage tiré d'Euthyphron de Platon qu'un pelatès ( «ouvrier») qui était au service de l'Athénien Euthyphron est mort quand il travaillait sur la terre de ce dernier et de son père à $\operatorname{Naxos}^{18}$. Le verbe $\dot{\theta} \theta \dot{\eta} \tau \varepsilon v \varepsilon v$ («travailler/ être aux gages de quelqu'un»), qui désigne dans le texte le statut du pelatès, renvoie à la pratique de mettre en gage les services personnels en garantie d'une dette jusqu'à son remboursement ${ }^{19}$, pratique dont l'Athènes classique fournit plusieurs exemples ${ }^{20}$. Or, le texte de Platon ne permet pas de dire si cette pratique faisait aussi partie du droit naxien; étant donné qu'une clérouquie athénienne s'était installée sur l'île vers le milieu du $\mathrm{v}^{\mathrm{e}}$ siècle ${ }^{21}$, les mots d'Euthyphron pourraient faire référence à la seule pratique athénienne.

Dans d'autres cas, il est plus facile de se demander si des relations d'indépendance ou d'interdépendance se sont développées entre les îles et Athènes. Il suffit de rappeler brièvement quelques témoignages déjà mis en valeur par les chercheurs modernes. En premier lieu, les horoi (bornes) que l'on a mis au jour dans les Cyclades portent les mêmes termes que ceux d'Athènes pour désigner la sûreté réelle. En font preuve les horoi découverts à Amorgos et à Naxos (IV siècle ou III $^{\mathrm{e}}$ siècle) dont les termes

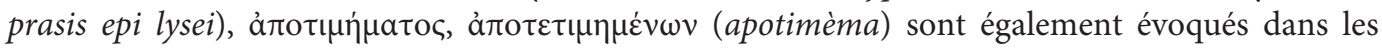
horoi attiques ${ }^{22}$. Parmi ces pratiques, l'institution de la prasis epi lysei (vente sous condition de

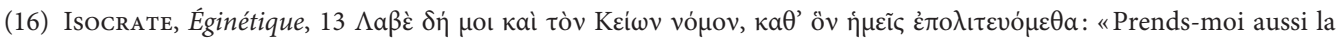
loi de Kéos qui réglait notre statut de citoyens» (trad. G. Mathieu, E. BRÉmond). Selon le plaideur siphnien qui tente de répondre à la revendication de son héritage, Thrasylochos l'a adopté conformément à la loi qui ordonne de prendre des égaux comme enfants adoptifs. Pour cela, il présente comme preuve la loi de Kéos qui règle le statut de citoyens. On ne dispose pas de détails sur cette loi.

(17) Voir Rubinstein 2003, p. 105. La difficulté de savoir si les exemples non attiques qui présentent des similitudes avec le système de justice athénien reflètent les réalités procédurales locales ou sont influencés par celles qui existent à Athènes, notamment quand les Athéniens y sont impliqués, est aussi soulignée par Low 2007, p. 85 n. 36.

(18) Platon, Euthyphron, 4c. Sur le statut de pelatès, voir Harris 2006, p. 261-262.

(19) Il faut distinguer entre cette pratique et la servitude pour dettes. Voir à ce propos, Harris 2006, p. 250-255.

(20) Harris 2006, p. 256-261, réunit et analyse ces exemples: Aristophane, Nuées, v. 240-241; Euripide, Alceste, v. 6-7; Hypéride, Contre Athénogène, 5-12; MénAndre, Héros, v. 16-38.

(21) Sur la clérouquie athénienne à Naxos, voir Bonnin 2015, p. 140-142, 171-173, avec des références bibliographiques.

(22) Ainsi, voir pour le terme désignant l'hypothèkè: à Athènes: Finley 1985, nº 1-7/ à Arkésinè d'Amorgos: FinLey 1985, nº 8 (IG XII, 7, 58); pour l'expression prasis epi lysei: à Athènes: Finley 1985, nº 11-101 / à Arkésinè d'Amorgos: FINLEY 1985, n 102 (IG XII, 7, 55): même si ce texte ne mentionne pas le terme horos, il est inclus par FINLEY dans sa liste d'horoi - il pourrait cependant être classé dans les actes de vente, voir à ce propos, GAME 2008, p. 145; pour le terme apotimèmaen ce qui concerne les pupilles: à Athènes: Finley 1985, nº 116-129 / à Arkésinè d'Amorgos: Finley 1985, $\mathrm{n}^{\circ} 130$ (IG XII, Suppl. 331)/ à Naxos: Finley 1985, nº 131 (IG XII, Suppl. 194); pour le terme apotimèma dotal: à Athènes: 
rachat) est aussi attestée dans des actes de vente des Cyclades: on lit l'expression érì $\lambda u ́ \sigma \varepsilon ı ~ d a n s$ un acte de vente de la fin du $\mathrm{IV}^{\mathrm{e}}$ siècle-début du $\mathrm{III}^{\mathrm{e}}$ siècle, trouvé près d'Arkésinè d'Amorgos ${ }^{23}$, ainsi que le verbe $\pi \rho i ́ a \mu \alpha$ dans un registre des ventes $\mathrm{du} \mathrm{IV}^{\mathrm{e}} / \mathrm{III}^{\mathrm{e}}$ siècle de Ténos ${ }^{24}$; les deux termes constituent le formulaire caractéristique des horoi attiques qui concernent cette institution. En effet, l'étude de la prasis epi lysei à travers ses attestations dans le monde grec a conduit aux conclusions que cette pratique pouvait varier d'une région à l'autre, mais qu'elle a eu «manifestement sa place sur l'aire de l'ancien empire athénien, même si les témoignages subsistants sont largement postérieurs à son apogée » ${ }^{25}$. Du côté du régime dotal et successoral des Cyclades, d'autres études se proposent de voir des pratiques identiques entre celui-ci et le droit attique et de les attribuer peutêtre à «une importation liée à la situation hégémonique d'Athènes pendant deux siècles ${ }^{26}$, sans manquer de citer en même temps les différences que l'on observe. Ainsi, et à titre d'exemple, un registre de constitution de dots de Ténos, malheureusement mutilé, indique que les biens dotaux

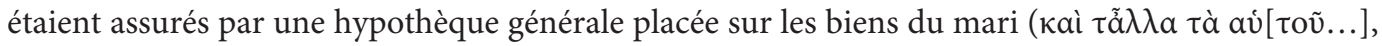

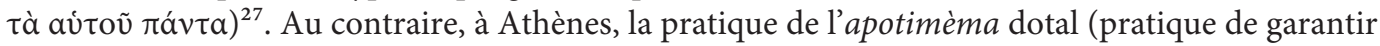
la dot de la femme en mettant en gage les biens du mari) semble avoir été moins lourde, puisque l'hypothèque était proportionnée à la $\operatorname{dot}^{28}$. De même, la pratique du consentement ou de la participation de la femme à l'aliénation des biens du mari était inconnue à Athènes, mais elle est attestée à Amorgos, à Ténos et à Délos ${ }^{29}$.

\section{LES TÉMOIGNAGES ÉPIGRAPHIQUES SUR LE DISPOSITIF JUDICIAIRE DES CYCLADES}

\section{A. Présentation des sources}

\section{Arkésinè d'Amorgos}

a. IG XII, 7, 3 (première moitié du IV siècle). Décret d'Arkésinè d'Amorgos.

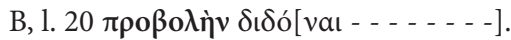

«qu'on [porte?] une probolè [...].

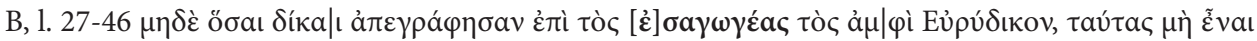

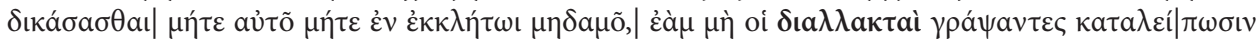

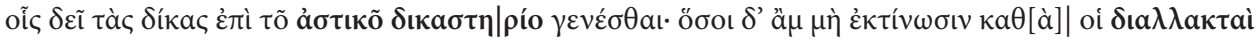

Finley 1985, n 132-153 / à Arkésinè d'Amorgos: Finley 1985, nº 154 (IG XII, 7, 56), nº 155 (IG XII, 7, 57) / à Naxos: FINLEY 1985, nº 156 (IG XII, Suppl. 195) / à Délos: nous n'avons aucune trace des horoi mentionnant l'apotimèma dotal et l'on ne peut donc pas savoir si cette pratique était ignorée ou si elle se traduisait par une manière autre que l'érection des horoi, voir sur ce sujet, VIAL 1985, p. 71 et n. 89. Sur ces termes, voir Harris 2006, p. 163-239; id. 2013b, p. 128-132.

(23) IG XII, 7, 55, 1. 14. Voir GAME 2008, p. 144-145, ainsi que la note précédente sur son classement.

(24) IG XII, 5, 872. ÉtIENne 1990 date l'inscription de la fin du IV e siècle, mais une date plus basse a été proposée. Voir GAME 2008, p. 105.

(25) Voir GAME 2008, p. 168-169: «sa diffusion au IV siècle est peut-être davantage une conséquence de l'évolution des procédures et des cadres juridiques, en ce qui concerne les prêts notamment, à cette époque».

(26) Voir Étienne 1990, p. 67 et n. 58.

(27) $I G$ XII, 5, 872, 1. 6, 10.

(28) Voir Étienne 1990, p. 63, 68. Pour une analyse du régime dotal et successoral à Ténos, voir id. 1990, p. 62-64, 67-68.

(29) Arkésinè d'Amorgos: IG XII, 7, 55, 1. 3-4 (fin IV /début III ${ }^{\mathrm{e}}$ siècle): Hègékratè participe à l'aliénation de propriétés

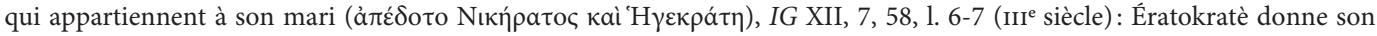

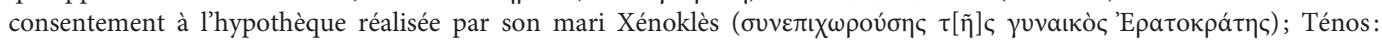
IG XII, 5, 872, 1.118 (IV/III ${ }^{\mathrm{e}}$ siècle): Mantô donne son consentement quand son mari, acheteur de terrains, procède à la résiliation de cette opération ( $\sigma v v \chi \omega \rho o v ́ \sigma \eta \varsigma ~ \kappa a i ̀ ~ \sigma v v \varepsilon \pi a ı v o u ́ \sigma \eta \varsigma ~ M a v \tau \tilde{\omega} \varsigma$ ); Délos: plusieurs inscriptions datant d'à partir du III $^{\mathrm{e}}$ siècle: voir le tableau récapitulatif de VIAL 1985, p. 70. 


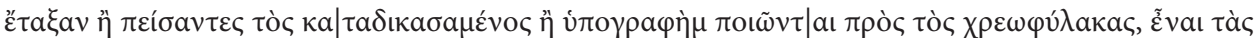

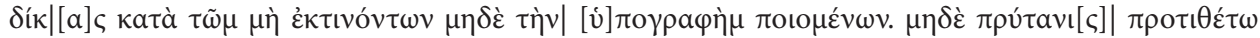

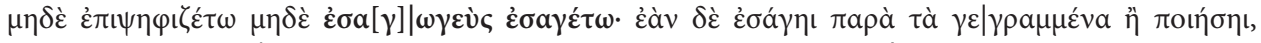

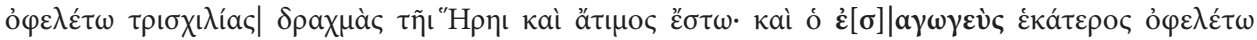

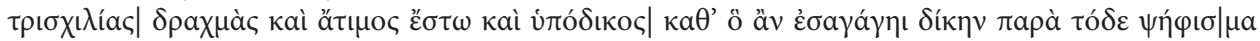

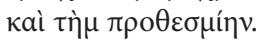

«Pour toutes les actions dont la demande a été déposée devant les eisagôgeis qui sont en fonction avec Eurydikos, qu'elles ne puissent pas donner lieu à jugement ni ici ni dans une cité appelée à juger, en quelque endroit que ce soit, à moins que les diallaktai, après les avoir inscrites, ne les transmettent à qui de droit pour qu'elles viennent devant l'astikon dikastèrion. Ceux qui n'acquitteront pas les sommes conformément aux décisions des diallaktai ou en persuadant (par composition/en négociant avec) leurs adversaires ou qui ne feront pas inscrire leur reconnaissance auprès des chréôphylakes, qu'ils soient exposés à une action pour non-paiement ou non-reconnaissance de dette. Que le prytane ne mette pas en délibération, qu'il ne mette pas aux voix, que l'eisagôgeus n'introduise pas (contrairement à ce qui est décidé); s'il introduit (un procès) ou s'il agit contrairement à ce qui est écrit, qu'il soit redevable d'une amende de trois mille drachmes consacrées à Héra et qu'il soit frappé d'atimie. Et que chaque eisagôgeus soit redevable d'une amende de trois mille drachmes et qu'il soit frappé d'atimie et qu'il soit soumis à une action en justice pour avoir introduit un procès contrairement au présent décret et à la prescription.» (trad. P. GAUThier, légèrement modifiée, à cause du problème posé aux lignes 34-35; cf. DreHer 1995, p. 147 n. 189).

b. IG XII, 7, 62 (seconde moitié du IV siècle) ${ }^{30}$. Syngraphè d'Arkésinè d'Amorgos.

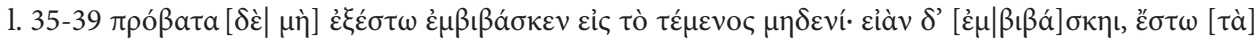

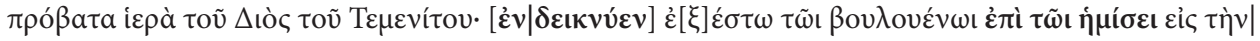

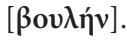

«Et qu'il ne soit permis à personne d'introduire du petit bétail sur le sanctuaire; si quelqu'un en introduit, que ce petit bétail soit propriété sacrée de Zeus Téménitès; que toute personne le désirant puisse aller accuser le contrevenant auprès du Conseil pour la moitié.» (trad. Chr. Chandezon, légèrement modifiée).

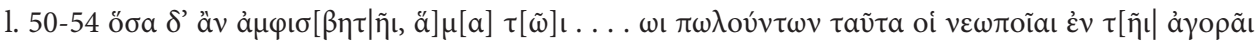

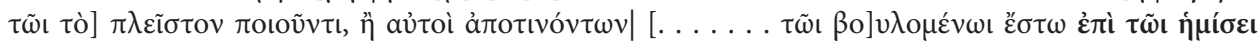

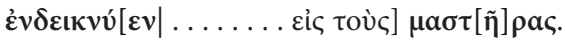

«Tout ce qui est sujet à contestation, que les néopes eux-mêmes le mettent en vente sur l'agora au plus offrant en présence de [...] ou qu'ils payent [...]; que toute personne le désirant puisse aller accuser le contrevenant pour la moitié [...] devant les mastères.» (trad. Chr. Chandezon, légèrement modifiée).

2. Délos ${ }^{31}$. Comptes des hiéropes.

a. IG XI, 2, 199 (273).

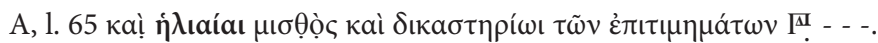

«pour l'Héliée et pour le tribunal des épitimètes, salaire [...] drachmes.

b. IG XI, 2, 203 (269).

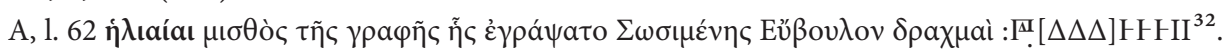
"pour l'Héliée, salaire pour le procès que Sosiménès a intenté à Euboulos cinq cents (?) oboles».

c. IG XI, 2, 223 (262).

A, 1.7 ì ııáaı $\mathrm{H}$.

«pour l'Héliée, (salaire) cent drachmes».

(30) Voir Chandezon 2003, p. 143, n 34; R \& O, p. 282-287, n 59.

(31) Pour ces quatre attestations, voir VIAL 1985, p. 147.

(32) Sur cette somme, voir VIAL 1985, p. 153 et n. 31. 
d. ID $442(179)$.

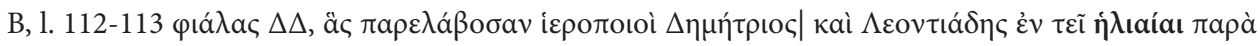

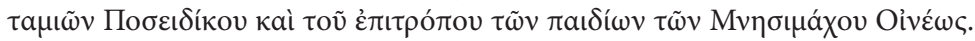

«20 phiales, qu'ont reçues les hiéropes Dèmètrios et Léontiadès dans l'Héliée, de la part des trésoriers, Poseidikos et le tuteur des jeunes enfants de Mnèsimachos, Oineus.» (trad. J.-Ch. MoretTr).

3. Ios

IG XII, 5, $2\left(\mathrm{IV}^{\mathrm{e}} / \mathrm{III}^{\mathrm{e}}\right.$ siècle $\left.^{33}\right)$. Règlement sacré d'Ios.

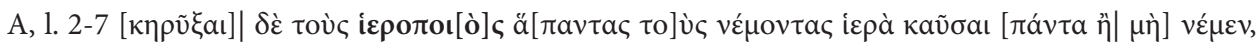

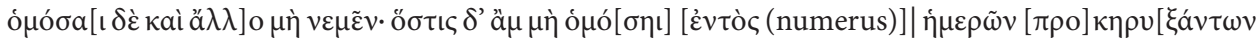

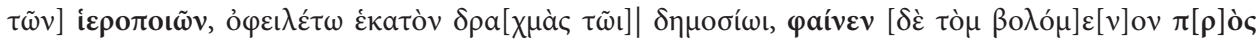

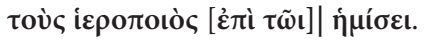

"Que les hiéropes fassent proclamer que tous ceux qui en font pâturer doivent faire marquer au feu tous (les bestiaux) comme sacrés ou ne pas les faire pâturer; qu'ils jurent ou qu'ils n'en laissent pas pâturer d'autres; que celui qui n'aura pas prêté serment sous [...] jours après la proclamation des hiéropes soit redevable d'une amende de cent drachmes à la caisse publique; et qu'il soit possible à celui qui le désire d'aller le dénoncer devant les hiéropes en touchant la moitié (de l'amende).» (trad. Chr. Chandezon, légèrement modifiée).

\section{Kéos}

IG $\mathrm{II}^{2}, 1128$ (milieu du IV $\mathrm{e}^{\mathrm{e}}$ siècle ${ }^{34}$ ). Inscription conservant les décrets de Karthaia, Korèsia et Ioulis sur le monopole athénien de l'ocre de Kéos.

Korèsia :

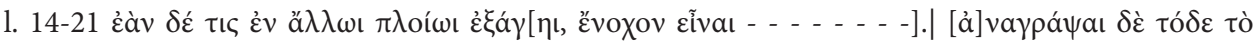

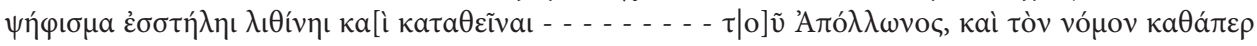

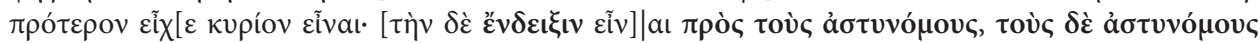

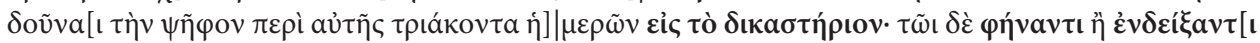

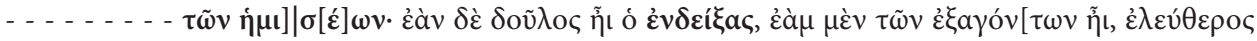

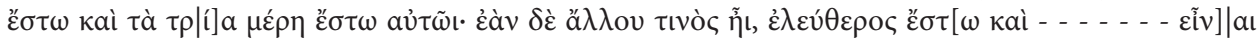

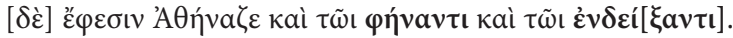

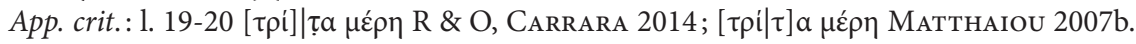

«Si quelqu'un exporte sur un autre navire, qu'il soit passible de [...]. Que l'on transcrive ce décret sur une stèle de marbre et qu'on l'installe [...] d'Apollon, et que la loi soit valable comme elle était dans le passé. Que l'accusation soit portée devant les astynomes, et que les astynomes introduisent l'affaire devant le tribunal dans un délai de trente jours. Pour le dénonciateur ou l'accusateur [...] de la moitié. Si l'accusateur est un esclave, s'il l'est des exportateurs, qu'il soit affranchi et qu'il reçoive un tiers (?). S'il est esclave d'un autre, qu'il soit affranchi et [...]. En outre, que l'ephesis ait lieu à Athènes tant pour le dénonciateur que pour l'accusateur.».

Ioulis:

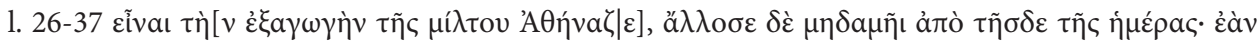

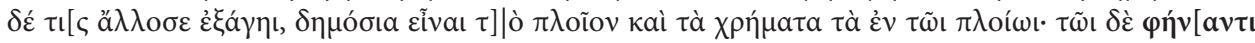

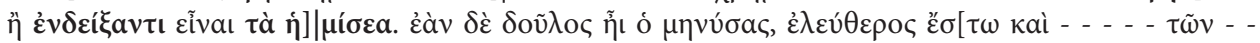

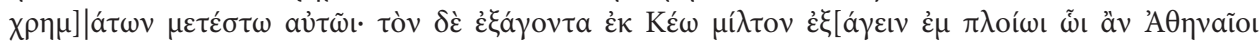

(33) Les $I G$ choisissent la datation du IV siècle, qui est fondée sur les lettres, tandis que Chandezon 2003, p. 140, n 32, préfère celle du $\mathrm{III}^{\mathrm{e}}$ siècle.

(34) La datation est approximative. Elle repose sur la forme des lettres et sur l'identification d'un des noms de la dernière ligne de l'inscription, sans que l'on puisse, pourtant, établir un contexte historique plus précis. Voir CargILL 1981, p. 138; Brun 1989, p. 123; R \& O, p. 207-208, n 40. CARrara 2014, p. 296-297, penche pour une datation peu après 362, sur la base de la différence de traitement entre les cités, comme le décret le laisse transparaître. Cf. aussi MAtTHAIOU $2007 \mathrm{~b}, \mathrm{n}^{\circ} 5$. 


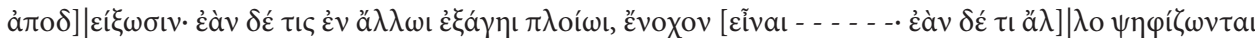

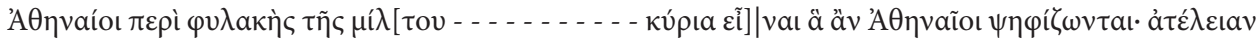

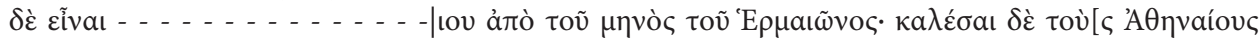

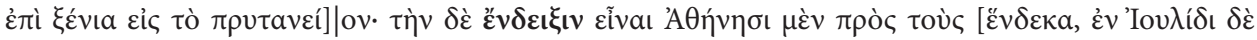

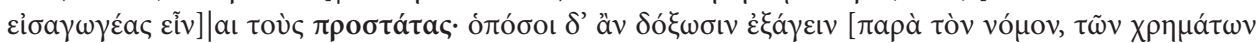

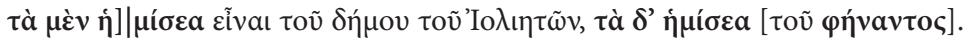

"Que l'exportation de l'ocre se fasse vers Athènes et nulle part ailleurs à partir de ce jour. Si quelqu'un exporte ailleurs, que le navire et la cargaison soient confisqués. Que le dénonciateur ou l'accusateur reçoive la moitié. Si l'accusateur est un esclave, qu'il soit affranchi et qu'il reçoive [...] de l'argent. Que celui qui exporte de l'ocre de Kéos le fasse sur un navire que les Athéniens auront indiqué. Si quelqu'un exporte sur un autre navire, qu'il soit passible de [...]. Si les Athéniens décident autre chose sur la sécurité de l'ocre [...], que ce que les Athéniens auront décidé soit valable. Qu'il $\mathrm{y}$ ait une exemption de taxes [...] à partir du mois d'Hermaion. Que l'on invite au prytanée les Athéniens pour le repas d'hospitalité. Que l'accusation se fasse à Athènes devant les Onze; à Ioulis, que les magistrats responsables pour l'introduction de l'affaire soient les prostatai. Tous ceux qui sembleront exporter contrairement à la loi, que la moitié de leurs biens soit confisquée au bénéfice du peuple d'Ioulis et que l'autre moitié appartienne au dénonciateur.».

\section{Naxos}

$I G \mathrm{II}^{2}, 179$ (second quart du IV $\mathrm{IV}^{\mathrm{e}}$ siècle ${ }^{35}$ ). Inscription concernant Athènes et Naxos.

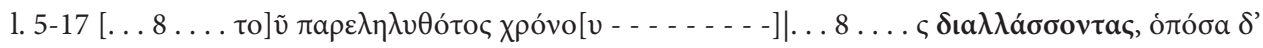

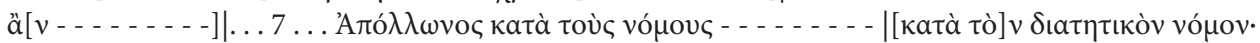

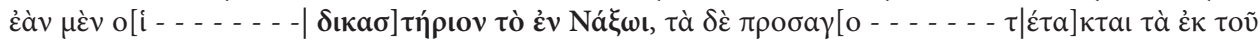

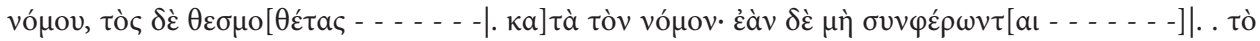

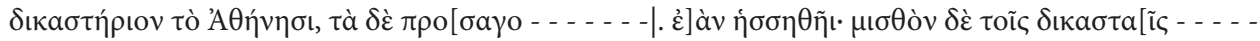

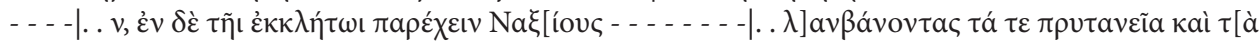

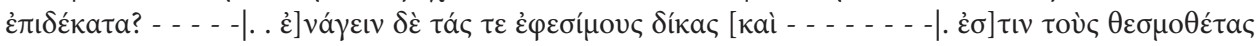

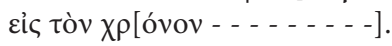

«[...] le temps passé [...] en conciliant, tout ce qui [...] d'Apollon selon les lois [...] selon la loi d'arbitrage. Si [...] le tribunal à Naxos, que ce qui est introduit (?) [...] par la loi, et que les thesmothètes $[\ldots]$ selon la loi. S'ils ne s'accordent pas [...] le tribunal à Athènes, et que ce qui est introduit (?) [...], s'il perd. Misthos pour les juges [...], et qu'à la cité appelée à juger (ekklètos) les Naxiens offrent (?) [...] recevant les frais de justice et [...]. Qu'on introduise les procès ephesimoi $[\ldots]$ les thesmothètes $[\ldots] .{ }^{36}$.

\section{Paros}

IG XII, 5, 108 (fin du ve siècle) ${ }^{37}$. Règlement relatif à la protection des arbres autour des sanctuaires de Paros.

(35) On lui attribue une date correspondant au second quart du Iv ${ }^{\mathrm{e}}$ siècle, mais des doutes subsistent quant à une datation plus précise. On propose trois datations soit vers 376/5 (suite à l'entrée "probable» de Naxos dans la Confédération) soit vers 360 (en raison des troubles dans la Confédération: voir CATALDI 1979, p. 12) soit vers 350 (en raison du rétablissement des bonnes relations entre Athènes et les cités qui lui sont restées fidèles pendant la guerre des Alliés: voir GAUTHIER 1972, p. 168; Reger 2004, p. 761). Pour un résumé de ces datations, voir Gauthier 1972, p. 168.

(36) Malheureusement, une traduction satisfaisante n'est pas possible, à cause de l'état très fragmentaire de l'inscription, qui fait que les difficultés de lecture, de restitution et d'interprétation abondent. Toutefois, il est clair que le décret fait allusion à une réconciliation, à une loi d'arbitrage, à l'existence d'un tribunal à Naxos, d'un tribunal à Athènes, à une cité ekklètos et à l'introduction des procès ephesimoi. CATALDi 1979, p. 9, propose les restitutions suivantes pour les 1. 8-9 o[i

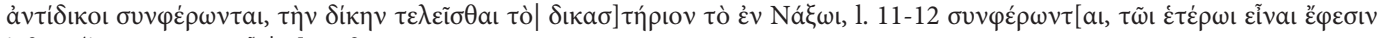

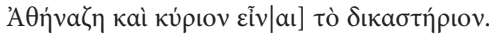

(37) Voir aussi LSCG, p. 205-206, $\mathrm{n}^{\circ} 111$; Inschriftliche, p. 217-219, $\mathrm{n}^{\circ} 58$. 


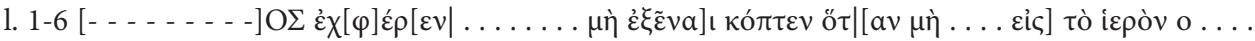

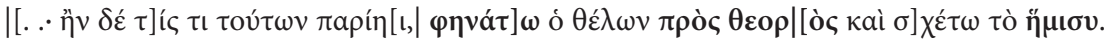

«[...] qu'il ne soit pas permis de couper quand [...] dans le sanctuaire [...]. Si quelqu'un transgresse un de ces règlements, que toute personne qui le veut puisse dénoncer (le contrevenant) devant les théores et qu'il reçoive la moitié.».

\section{Siphnos}

Décret d'Athènes à propos de Siphnos.

a. Première édition et datation: Woodhead 1957, p. 231, nº 87; Agora XVI, $50\left(360-350^{38}\right)$.

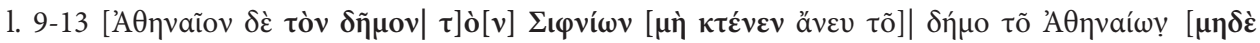

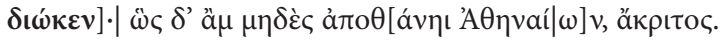

"Que le peuple des Siphniens ne soit pas autorisé à exécuter ou à poursuivre un Athénien sans

l'accord du peuple des Athéniens; pour qu'aucun des Athéniens ne meure sans jugement.».

b. Nouvelle édition et datation: Matthaiou 2010 (fin du ve siècle ${ }^{39}$ ).

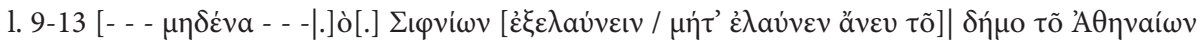

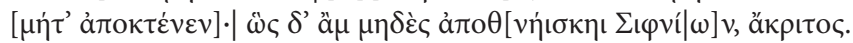

"Qu'aucun [...] des Siphniens ne soit autorisé à bannir ou à exécuter sans l'accord du peuple des

Athéniens; pour qu'aucun des Siphniens ne meure sans jugement.».

8. Théra

IG XII, 3 suppl., 1289 (IV siècle). Inscription de Théra ${ }^{40}$.

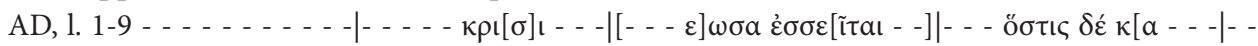

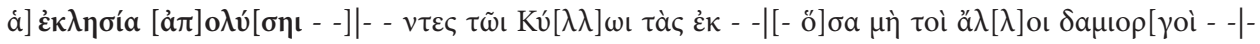

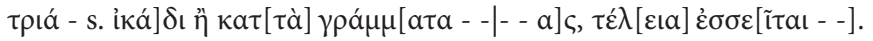

«[... jugement (?) [...] il sera [...] celui que l'Assemblée acquittera $[\ldots]$ Kyllos $[\ldots]$ tout ce que les autres démiurges ne $[\ldots]$ le vingtième jour ou d'après les règles écrites (?) [...] sera accompli (?) $[\ldots] »$.

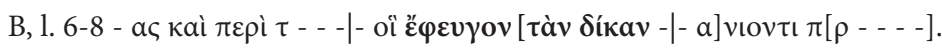

«[...] ceux qui étaient accusés $[\ldots] »$.

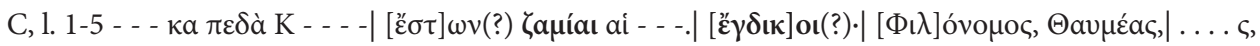
Nвóvıкос,"Н

«[...] entrave [...] que soient (?) comme peines celles que [...] ceux qui poursuivent en justice (?); Philonomos, Thaumeas, [...], Néonikos, Hèrippos».

\section{B. Discussion des témoignages}

\section{Les procédures attestées}

\section{Endeixis}

Dans l'état actuel de nos connaissances qui viennent des cas attestés à Athènes, l'endeixis était une procédure selon laquelle un citoyen, qui accusait une personne devant le magistrat compétent (thesmothètes, Onze, basileus), avait la possibilité de procéder par la suite à son arrestation. Cette

(38) Sur cette date, voir Woodhead 1957, p. 231. Par conséquent, ce décret entrait dans la discussion autour de la politique athénienne au sein de la seconde Confédération maritime. Voir GaUthier 1972, p. 169 n. 2; CARGILL 1981, p. 137.

(39) La nouvelle datation tient compte de la forme des lettres et aussi d'une nouvelle lecture qui conduit à une nouvelle restitution et interprétation des 1. 10-13, dont le contenu convient à la fin du ve siècle plutôt qu'au milieu du IV siècle. Voir Matthaiou 2010, p. 52-53.

(40) Il s'agit du seul décret du IV siècle conservé par l'île. Voir Nigdelis 1990, p. 74 n. 5. Au contraire, l'inscription n'est pas citée dans la liste des décrets des cités grecques établie par RHODEs, Lewis 1997, p. 296-297. 
procédure s'appliquait aux infractions suivantes: lorsque l'accusé avait commis des infractions contre la propriété, qu'il avait exercé ses droits civiques, alors qu’il en était privé, qu'il était entré dans des lieux interdits, alors qu'il était soupçonné ou accusé d'homicide, ou encore qu'il était rentré d'exil sans y être autorisé ${ }^{41}$. Dans les Cyclades, le terme est attesté dans la syngraphè (qui correspondait plus ou moins à un bail agricole) de la cité d'Arkésinè d'Amorgos (1b), qui règle les conditions d'affermage d'une terre sacrée appartenant à Zeus Téménitès. Même si dans la première clause que nous avons citée, le terme $\dot{\varepsilon} \nu \delta \varepsilon เ \kappa v u ́ \varepsilon v$ est restitué (1. 37-38), cette restitution semble assurée, puisque le même terme est attesté par la suite dans un contexte similaire (1. 53). Dans la première occurrence, on voit que toute personne qui le veut peut porter une accusation par voie d'endeixis contre celui qui permet l'entrée du petit bétail dans le téménos de Zeus Téménitès (l. 36-38). L'accusation est déposée devant le Conseil d'Arkésinè (le mot est restitué), ce qui, faute d'autres précisions, nous conduit à nous demander s'il est le seul organe qui rendra la justice (l. 39). Au cas où l'accusé est reconnu coupable, la moitié de la somme obtenue par la confiscation des biens est remise à l'accusateur. Dans la seconde occurrence, l'indemnité reçue par l'accusateur est fixée dans les mêmes termes, mais cette fois l'accusation est portée contre les néopes, qui constituent un collège compétent pour gérer la terre sacrée, et elle est déposée auprès des mastères ${ }^{42}$ (1. 50-54). L'inscription ne précise pas si la pratique de l'arrestation de l'accusé par l'accusateur avait cours à Arkésinè, mais elle fournit une information qui différencie l'endeixis athénienne de celle d'Arkésinè, où l'accusateur pouvait recevoir la moitié de la somme acquise par la vente du bien, au cas où l'accusé serait reconnu coupable ${ }^{43}$.

On trouve les mêmes conditions concernant l'indemnité pour l'accusateur dans l'île de Kéos, dans l'inscription fixant l'organisation du monopole athénien de l'ocre de Kéos (4). En ce qui concerne les deux cités, Korèsia et Ioulis, on peut lire les réglementations judiciaires qui sont fixées pour les contrevenants aux accords passés. La procédure d'endeixis ne concerne plus une infraction de caractère religieux, mais une affaire commerciale. Dans le cas de Korèsia, la procédure judiciaire a lieu, dans un premier temps, devant les magistrats locaux, les astynomes, qui introduisent par la suite l'affaire devant le tribunal dans un délai de trente jours, au cas où quelqu'un exporte de l'ocre sur un navire, excepté celui que les Athéniens ont indiqué (1. 16-18). La moitié de la somme qui résulte de la condamnation est remise tant au dénonciateur ( $\varphi \eta ́ v \alpha v \tau$ ) qu'à l'accusateur ( $\dot{\varepsilon} \delta \varepsilon \dot{\xi} \xi \alpha \nu \tau)$ (1. 18-19). En second lieu, la personne qui fait la dénonciation ( $\varphi \eta ́ v a v \tau \iota)$ ou porte l'accusation ( $\dot{\varepsilon} \nu \delta \varepsilon \dot{\xi} \xi \alpha \nu \tau \iota)$ contre le contrevenant a le droit d'exercer l'ephesis à Athènes (1. 21) $)^{44}$. Dans le cas d'Ioulis, on trouve, en premier, une clause judiciaire qui fait référence au dénonciateur ( $\varphi \eta ́ v a v \tau \iota)$ ou à l'accusateur ( $\dot{\varepsilon} v \delta \varepsilon \dot{\xi} \xi \alpha v \tau)$ de la personne qui exporte de l'ocre ailleurs qu'à Athènes, et à la récompense qu'il reçoit, c'est-à-dire la moitié de la somme obtenue par la confiscation du navire et de la cargaison (1. 28-29). Il est prescrit, par la suite, que, si quelqu'un exporte sur un autre navire que celui que les Athéniens ont indiqué, il sera accusé, mais le décret ne donne pas de renseignements supplémentaires (1. 30-31). Suit, quelques lignes plus loin, une nouvelle clause qui

(41) Voir, à titre indicatif, Aristote, Constitution d'Athènes, LII. 1; [DÉmosthène], Contre Nicostratos, 14; Démosthène, Contre Théocrinès, 1, 14. Voir sur cette procédure, Hansen 1976.

(42) Sur les mastères, voir ci-après.

(43) La moitié de l'amende imposée au coupable sera aussi reçue par la personne qui portera une accusation par endeixis à Ierapytna de Crète dans une affaire à caractère militaire: IC III, iii, 1, 1. 7-9 (III siècle). Pour l'attestation du terme dans d'autres régions du monde grec, voir: Cos: IG XII, 4, 1: 132, A, b, 1.49 (ca. 300); Kymè en Éolide: IK Kyme 13, vi, 1. 89-90 (II ${ }^{\mathrm{e}}$ siècle); Stymphalos en Arcadie: IG V, 2, 357, 1. $89-90$ (III ${ }^{\mathrm{e}}$ siècle); Thasos: SEG 17.415, 1. 1, 5 (IV ${ }^{\mathrm{e}}$ siècle).

(44) Sur ce terme dans les documents attiques du Ive siècle, voir Pelloso 2016, p. 37-42. 
stipule que l'accusation ( $\left.\varepsilon^{v} v \delta \varepsilon \xi \xi v\right)$ se fera à Athènes auprès du collège des Onze, tandis qu'à Ioulis les prostatai ${ }^{45}$ sont les magistrats chargés de l'affaire $(1.35-36)^{46}$.

Phasis

On voit que la même inscription fait référence à la procédure de phasis ${ }^{47}$, qui est attestée pour les mêmes cas que ceux concernés par l'endeixis: on y ajoute une dernière clause du décret d'Ioulis qui stipule que, si quelqu'un viole une des clauses de la loi qui concerne les exportations, le dénonciateur (?) ( $\varphi \eta ́ v a v \tau$, le mot est restitué) recevra la moitié de la somme obtenue grâce à la confiscation de la cargaison (1.36-37).

Le fait que les deux décrets, de Korèsia et d'Ioulis, diffèrent aussi bien pour l'ensemble des clauses qu'en ce qui concerne les clauses judiciaires s'explique en partie par le fait que, dans le cas de Korèsia, on avait déjà voté d'autres décrets sur l'ocre (1. 16) et en partie par le comportement différent de ces deux cités envers Athènes ${ }^{48}$. Deux épisodes de révolte contre la seconde Confédération ont eu lieu dans l'île; le premier date de 364/3, peut-être à l'occasion de la présence de la flotte thébaine en Égée ${ }^{49}$, et concerne toutes les cités de l'île; le second, de 363/2, concerne seulement la révolte d'Ioulis ${ }^{50}$. De ce fait, si la présence d'Athènes peut suggérer qu'elle intervient dans les affaires de Kéos, il ne faut pas ignorer que cette intervention a eu lieu après une révolte et ne dit rien des procédures trouvées à Kéos: en dépit des similitudes qu'elles présentent avec celles d'Athènes (voir pour la phasis: infractions relatives au commerce, introduction de l'affaire devant un tribunal à Korèsia, amende imposée), elles conservent leurs propres caractéristiques (voir pour l'endeixis: infraction commerciale et amende imposée; noms et compétences des magistrats locaux; dispositions pour les esclaves ${ }^{51}$ ).

Quant à Paros, l'inscription qui porte le règlement relatif à la protection des arbres autour

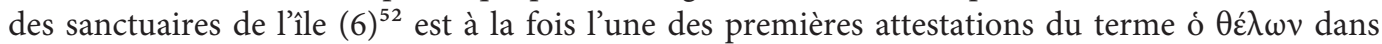
le monde grec hors d'Athènes ${ }^{53}$ et l'une des premières attestations (le mot est restitué, mais la restitution est très plausible $)^{54}$ dans les sources disponibles de la procédure de phasis. D’après

(45) Sur les prostatai, voir ci-après.

(46) L'ordre dans lequel les clauses de ces deux décrets sont transcrites n'est pas bien organisé, ce qui soulève parfois des questions à propos des différentes étapes des procédures qui y sont décrites, notamment en ce qui concerne le cas d'Ioulis. À titre d'exemple, il est évident que le mot ephesis manque dans le décret d'Ioulis. Si on garde à l'esprit la manière dont les décrets ont été rédigés, on peut se demander si le cas d'Ioulis ne constitue pas un cas similaire à celui de Korèsia. Pourrait-on, par conséquent, distinguer deux étapes, celle devant les prostatai et celle devant les Onze, qui sont les magistrats chargés de l'introduction de l'affaire devant les tribunaux athéniens, très probablement dans les instances appelées à juger à Athènes? Le décret ne permet malheureusement pas de répondre de façon affirmative. Il semble que cette hypothèse soit aussi exprimée par Low 2007, p. 89. Des hypothèses différentes sont avancées par CATALDI 1979, p. 15-17; CARRARA 2014, p. 308.

(47) Voir l'emploi du terme phasis en tant que procédure judiciaire à: Astypalaia: IG XII, 3, 168, 1. 12 (II siècle); Cos: IG XII, 4, 1: 100, 1.28 (II ${ }^{\mathrm{e}}$ siècle); Magnésie du Méandre: Magnesia 4, 1. 35 (II siècle); Nisyros: IG XII, 3, 87, 1.9 (mot restitué) (III ${ }^{\mathrm{e}}$ siècle); Thasos: SEG 17.416, 1.19 (IV ${ }^{\mathrm{e}}$ siècle).

(48) Voir Cataldi 1979, p. 16-17; Vélissaropoulos 1980, p. 187; R \& O, p. 208, nº 40; Matthaiou 2007b, p. 48; Carrara 2014, p. 296-297.

(49) Diodore de Sicile, XV. 78. 4 - 79. 1.

(50) $I G \mathrm{II}^{2}, 111$. Autres éditions: R \& O, n 39; Matthaiou 2007a, $\mathrm{n}^{\circ} 2$. Sur les événements des années 364-362, voir BonNin 2015, p. 267-270.

(51) Sur ces dispositions, voir R \& O, p. 208-209.

(52) Pour des peines imposées à ceux qui coupent du bois dans un sanctuaire: $I G \mathrm{II}^{2}, 1362$ (Athènes); IG IX, 2, 1109 (Magnésie en Thessalie); IG V, 1, 1390 (Messénie).

(53) Voir, pour une liste complète, Rubinstein 2003, p. 88 n. 3.

(54) On dispose aussi d'une inscription à caractère religieux qui vient de Minoa d'Amorgos du ve/Ive siècle (IG XII, 7, 220), dont l'état très fragmentaire ne permet pas de savoir si elle fait référence à la procédure de phasis. Ainsi, les 1. 3-6

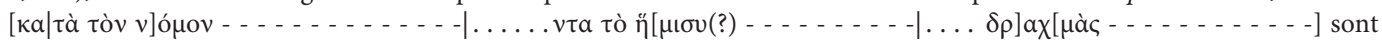


l'inscription, toute personne qui le veut pourra dénoncer $([\varphi \eta v a ́ \tau] \omega)$ devant les théores celui qui aura violé les règlements à propos des végétaux et des arbres autour des sanctuaires de l'île (1. 4-5). L'inscription ne précise pas si la dénonciation est introduite par les théores devant un tribunal - on ne sait rien des tribunaux à Paros - ou si ce sont les théores seuls qui vont prendre une décision. Elle atteste, pourtant, que le dénonciateur recevra la moitié de l'amende (?), au cas où l'accusé serait reconnu coupable (1. 6). L'inscription d'Ios (3), qui est assez fragmentaire, revêt aussi un caractère religieux. Le règlement sacré concerne cette fois les pâturages, mentionnant que leur utilisation n'était possible que par les animaux marqués d'un signe, des bêtes qui étaient alors considérées comme sacrées ${ }^{55}$. Suit une clause judiciaire qui stipule que toute personne qui le veut pourra dénoncer ( $\varphi$ aívعv) devant les hiéropes celui qui ne respectera pas ce que les hiéropes ont indiqué sur le pâturage des animaux sacrés (1. 5-6). Si ce dernier est reconnu coupable - cette inscription ne précise pas non plus l'organe qui jugera l'affaire -, le dénonciateur recevra la moitié de l'amende imposée au coupable (1. 6-7). Il en ressort donc que des amendes similaires seront imposées dans le cas d'une affaire d'impiété dans les deux îles, même si le type d'impiété n'est pas le même. Comme les sources conservées ne nous permettent pas de savoir quel type d'amende ou de peine était imposé dans les procès d'impiété initiés par la phasis à Athènes, ces deux témoignages qui viennent des Cyclades pourraient constituer une piste de réflexion. On est ainsi autorisé à se demander si le dénonciateur devait recevoir la moitié de l'amende imposée au coupable dans l'affaire d'impiété attestée dans une inscription athénienne du milieu du IV siècle (367-348?) portant sur une loi relative aux Mystères d'Eleusis ${ }^{56}$ : une dénonciation est prévue contre celui qui violera des règlements à propos de l'initiation aux Mystères, et indique que la dénonciation sera déposée auprès du basileus, qui l'introduira par la suite devant l'Héliée, mais la peine ou l'amende ne sont pas connues.

\section{Probolè}

Le décret d'Arkésinè d'Amorgos de la première moitié du IV siècle (1a) cite le terme $\pi \rho \circ \beta$ $\lambda \hat{\eta}$ (1. 20). Ce terme pourrait faire référence à la procédure de probolè, mais on n'est pas en mesure d'énumérer les cas dans lesquels elle aurait été appliquée et si elle était la même que la procédure athénienne, puisque le décret est trop fragmentaire pour arriver à une conclusion ferme en ce qui concerne le contexte politique précis dans lequel ont été prises les décisions qu'il contenait. Dans le cas de l'Athènes du IV e siècle, l'accusateur déposait sa plainte lors d'une session de l'Assemblée, soit contre les sycophantes, soit contre ceux qui avaient trompé le peuple par leurs promesses, parce qu'ils n'avaient pas tenu leurs engagements, soit contre ceux qui avaient transgressé la fête des Dionysies. L'Assemblée se prononçait par un vote et un procès pouvait suivre devant le tribunal populaire. Le vote à l'Assemblée et le procès devant le tribunal étaient deux étapes distinctes et la procédure de la probolè ne concernait que le vote $\mathrm{e}^{57}$.

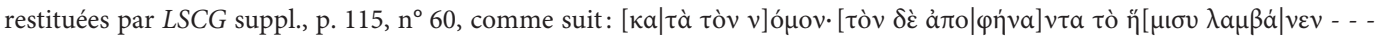

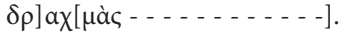

(55) Voir Chandezon 2003, p. 140-141, nº 32

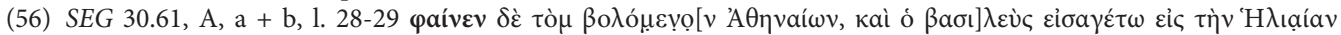
$\kappa \alpha[\ldots \ldots \ldots \ldots 26 \ldots \ldots \ldots \ldots$. dénoncer, et que l'archonte-roi introduise l'affaire devant l'Héliée [...] que le Conseil délibère pour lui, parce qu'il a commis une injustice.»

(57) Aristote, Constitution d'Athènes, XLIII. 5; Démosthène, Contre Midias, 9. Sur la procédure de probolè, voir HARRIS 2008, p. 79-80; id. 2013a, p. 213-214. 


\section{Les magistrats à compétences judiciaires}

\section{Astynomes}

À Korèsia de Kéos (4), les astynomes sont dotés de compétences judiciaires relatives au commerce (1. 17-18), mais le manque de sources laisse la question ouverte sur leurs autres fonctions. En revanche, les astynomes dans le registre des ventes du $\mathrm{IV}^{\mathrm{e}} / \mathrm{III}^{\mathrm{e}}$ siècle de Ténos sont chargés d'enregistrer ces actes ${ }^{58}$. Dans la Constitution d'Athènes, Aristote fait référence à l'existence de dix astynomes, dont cinq à Athènes et cinq au Pirée, qui veillent à la mise en ouvre de diverses dispositions visant au bon fonctionnement de la vie quotidienne de la cité ${ }^{59}$. À en juger par l'exemple de Korèsia, le manque de référence d'Aristote aux pouvoirs judiciaires des astynomes athéniens ne nous empêche pas d'énoncer l'hypothèse qu' «ils pourraient imposer, comme les autres magistrats, des amendes ou déférer en justice toute personne accusée d'avoir violé les dispositions qui étaient de leur ressort $»^{60}$.

\section{Diallaktai}

Dans la seconde partie du décret d'Arkésinè (1a), on cite le terme $\delta\left\llcorner\alpha \lambda \lambda \alpha \kappa \tau \alpha i^{61}(1.31,34)\right.$ et on inclut des réglementations judiciaires définissant le processus suivi pour les cas qui concernent très probablement des dettes (1. 33-37) ${ }^{62}$. La présence et l'intervention des diallaktai, c'est-à-dire des conciliateurs, dans la cité d'Arkésinè, et la prise de mesures pour résoudre les différends imminents et éviter toute aventure judiciaire plaident en faveur de l'hypothèse que des dissensions internes ont dû régner à Arkésinè pendant une période assurément antérieure au décret, mais qu'on ne peut pas dater plus précisément en raison de l'état très fragmentaire du sommet de l'inscription ${ }^{63}$. La période de crise et le recours à des mesures exceptionnelles, la présence des diallaktai conjointement avec

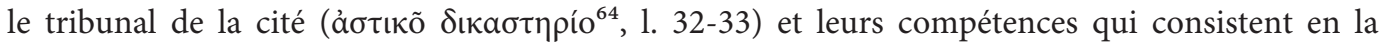
conciliation des parties et la prise d'une décision judiciaire pourraient former les caractéristiques des juges étrangers qui circuleront dans le monde grec surtout à partir de la fin du iv siècle ${ }^{65}$. L'inscription ne permet néanmoins ni d'assimiler les diallaktai d'Arkésinè à des juges étrangers ni d'obtenir des détails sur leur identité (origine, nombre).

(58) IG XII, 5, 872, 1. 1-2. Sur leurs compétences, voir Giannakopoulos 2009, p. 11-12. Outre Ténos, il existe des exemples dans des cités du monde grec de l'époque hellénistique (Histiée, Délos) qui montrent la variété des compétences des astynomes. Voir à ce propos, id. 2009, p. 9-19.

(59) Aristote, Constitution d'Athènes, L. 2.

(60) Voir MacDowell 1978, p. 159.

(61) Voir aussi l'emploi des termes $\delta\llcorner\alpha \lambda \lambda \alpha \gamma \eta \dot{~ e t ~} \delta\llcorner\alpha \lambda \lambda \alpha ́ \sigma \sigma \omega$ dans d'autres régions voisines, chacune présentant ses propres caractéristiques quant aux gens qui interviennent pour résoudre les conflits: 1 . Athènes: $I G \mathrm{II}^{2}, 10$ (voir aussi $\mathrm{R} \&$ $\mathrm{O}$, p. 20-27, $\mathrm{n}^{\circ} 4$ ): réconciliation entre les oligarques et les démocrates (années après la restauration de la démocratie en 403/2); 2. Naxos (5): IG $\mathrm{II}^{2}, 179$ : l'inscription fait allusion à une réconciliation (second quart du IV siècle); 3 . Paros: SEG 56.112 (= Crowther, Matthaiou 2004-2009, p. 31-38) (voir aussi R \& O, p. 146-149, n² 29): réconciliation entre les gens de Paros, grâce à l'intervention de la seconde Confédération maritime (372).

(62) Robert 2007, p. 314, y voit, d'un côté, des procès dans une cité entre concitoyens, mais, d'un autre côté, il met l'accent sur le fait que "citoyens dans la ville et bannis de retour forment deux groupes distincts, chacun bien constitué et avec des intérêts divergents». Il s'agirait donc de deux communautés. Il n'est cependant pas sûr que les procès soient dus au retour des bannis. Voir DreHER 1995, p. 147.

(63) Voir Gauthier 1972, p. 333-337; Nigdelis 1990, p. 30; Dreher 1995, p. 146-148.

(64) Nous trouvons souvent l'opposition entre le xenikon et le politikon dikastèrion. Voir Scafuro 2015, p. 366-367. Tandis que le mot ảotıkóc est souvent traduit comme «celui de la ville » par opposition à la chôra, le contexte de l'inscription (surtout la présence des $\delta\llcorner\alpha \lambda \lambda \alpha \kappa \tau \alpha i)$ nous permet de le traduire comme «tribunal de la cité». Voir GaUthiER 1972, p. 333; Dreher 1995, p. 147 n. 183; Arnaoutoglou 1998, nº 66.

(65) Sur les cas du dernier quart du Iv e siècle, voir Scafuro 2015, p. 365. Sur les juges étrangers et leur mission pendant l'époque hellénistique, avec des références à la bibliographie antérieure sur ce sujet, voir HAMON 2012, p. 195-222; Magnetto 2016, p. 28-34. 


\section{Eisagôgeis}

Les eisagôgeis, c'est-à-dire les magistrats ayant le pouvoir d'introduire les procès devant les tribunaux, sont évoqués à plusieurs reprises dans le décret d'Arkésinè (1a) (1. 18-19, 28, 39-40, 42-43). À Athènes, les eisagôgeis sont attestés au IV siècle, même si le terme se rencontre pour la première fois dans le décret de Thoudippos $(425 / 4)^{66}$, où les eisagôgeis étaient responsables des plaintes déposées par les cités alliées de la Ligue de Délos contre l'augmentation du phoros. La recherche moderne s'accorde pour souligner que ce corps n'est pas identique au collège des cinq eisagôgeis décrits par Aristote dans la Constitution d'Athènes et dont la responsabilité concernait les affaires dites emmènoi ${ }^{67}$. Il semble que, dans le décret d'Arkésinè, les eisagôgeis avaient la compétence étendue d'introduire tout type d'affaire dans le tribunal de la cité ${ }^{68}$. Leur importance peut même être soulignée indirectement si l’on étudie les sanctions qui leur seront imposées au cas où ils violeraient les clauses du décret sur l'introduction des procès (1. 41-46). Non seulement la syntaxe de la phrase lie les eisagôgeis aux prytanes, qui étaient responsables de l'introduction et du vote de propositions, mais l'inscription précise qu'ils devront, en cas de culpabilité, payer une amende de trois mille drachmes, seront frappés d'atimie et déférés en justice. Les amendes et les peines imposées semblent sévères mais se justifient en raison de la crise interne qui a éclaté à Arkésinè.

\section{Hiéropes}

Comme les hiéropes d'Athènes ${ }^{69}$, les hiéropes qui sont attestés dans l'inscription d'Ios (3) $(1.3,5,6)$ disposent des compétences judiciaires, à la différence que les hiéropes athéniens pouvaient juger les affaires dont l'amende ne dépassait pas un certain montant. Au contraire, l'inscription en question ne permet pas de savoir si ces hiéropes jugent toutes les phaseis ou si une restriction est imposée quant à la somme qu'ils peuvent régler: on pourrait, par exemple, se demander si la somme des cent drachmes due tombait dans le domaine de leur compétence (1. 5).

\section{Mastères}

On a vu que dans la syngraphè d'Arkésinè (1b), l'accusation qui est portée contre les néopes est déposée auprès des mastères (1. 54). La compétence des néopes en tant que collège chargé de gérer la terre sacrée semble justifier le dépôt de l'accusation auprès des mastères et non auprès du Conseil, comme c'était le cas de l'accusation portée contre celui qui permettait l'entrée du petit bétail dans le téménos de Zeus Téménitès (1. 36-38): loin de l'influence athénienne, où les magistrats contrôleurs s'appelaient euthynoi ${ }^{70}$, «mastères» est l'appellation donnée aux magistrats contrôleurs dans la cité d'Arkésinè $^{71}$ - comme à Delphes et dans des cités du Péloponnèse à quelques différences près ${ }^{72}-$, qui recueillent les plaintes contre les magistrats défaillants et qui, selon toute vraisemblance, sont aussi chargés de la reddition de comptes. L'inscription ne permet cependant pas de savoir si les

(66) $I G \mathrm{I}^{3}, 71,1.7,12,13,59-60$.

(67) Aristote, Constitution d'Athènes, LII. 2-3. Sur le terme emmènos, voir Bresson 2008, p. 117.

(68) Cf. Nigdelis 1990, p. 30. Voir aussi les eisagôgeis attestés en tant que magistrats à compétences judiciaires à: Éphèse: Ephesos 4, A, 1. 5-6 (III ${ }^{\mathrm{e}}$ siècle); Clazomènes et Temnos: Klazomenai 4, 1. 25, 40 (II siècle); Lampsakos: IMT NoerdlTroas 4, 1. 27 (II siècle).

(69) Voir, e.g., $I G \mathrm{I}^{3}, 82,1.25-28$ (421/0). Le décret oblige les hiéropes à imposer une amende allant jusqu'à cinquante drachmes pour tout comportement malséant pendant la procession. Si un individu est jugé redevable d'une amende supérieure, le procès sera jugé par le tribunal. Cf. MACDowell 1978, p. 235-236; BrUn 2005, p. 247-249, n 129; MAKRES 2014, p. 185-196, qui exprime l'hypothèse qu'il s'agit probablement d'un décret relatif à la fête des Thèseia et non à la fête des Hèphaisteia, comme on le pensait jusqu’à présent.

(70) Sur les euthynoi athéniens, voir PIÉRART 1971, p. 526-573.

(71) Sur les mastères d'Arksésinè, voir Nigdelis 1990, p. 31; Migeotte 2014, p. 71-72. Voir surtout Fröhlich 2004, p. 185-186.

(72) Voir FröHLICH 2004, p. 181-192. 
mastères jugent l'affaire ou s'ils l'introduisent devant un tribunal, et il serait préférable de ne pas en tirer de conclusions sur la base des sources attiques à propos des compétences des euthynoi.

\section{Prostatai}

Les prostatai ne sont pas attestés dans un cadre athénien. En revanche, ce sont les magistrats locaux à Ioulis de Kéos (4) qui sont chargés de l'endeixis ${ }^{73}$. Les sources conservées interdisent de savoir s'ils disposent d'autres compétences. Pour les Cyclades, cette magistrature est aussi attestée dans une inscription de Karthaia de Kéos de la fin du $\mathrm{III}^{\mathrm{e}}$ siècle ${ }^{74}$, qui ne précise rien de leur juridiction, ainsi que dans deux inscriptions de Ténos de l'époque hellénistique ${ }^{75}$, où il doit s'agir de magistrats chargés des fonctions politiques ${ }^{76}$.

\section{Les institutions à compétences judiciaires}

Boulè

Outre les décrets qui sont conservés dans les Cyclades et qui témoignent de l'existence d'un Conseil dans plusieurs îles ${ }^{77}$, la syngraphè d'Arkésinè d'Amorgos (1b) atteste aussi les fonctions judiciaires du Conseil de la cité ([ßou入ńv]) (1. 39), au cas où l'on se fierait à la restitution proposée. Comme nous l'avons déjà indiqué, il n'est pas sûr que ce Conseil juge l'affaire portée devant lui. On est ainsi autorisé à se demander s'il a le droit de juger les affaires dont l'amende ne dépasse pas un certain montant, comme c'est le cas du Conseil des Cinq Cents d'Athènes ${ }^{78}$, ou s'il introduit tous les procès au tribunal de la cité (1a).

\section{Dikastèria}

Sur la base des inscriptions disponibles, l'existence des tribunaux dans les Cyclades de l'époque classique est attestée de manière explicite dans les cités suivantes: à Arkésinè d'Amorgos (1a), le

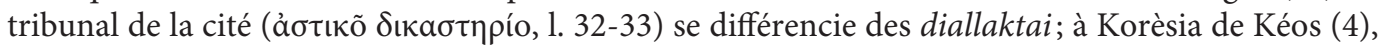

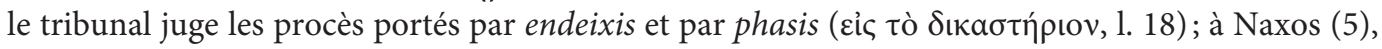

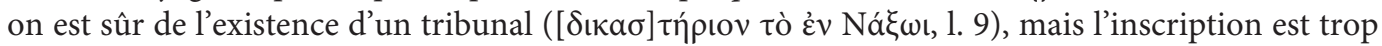
lacunaire pour tirer d'autres conclusions. À Ioulis de Kéos (4), le mot dikastèrion n'est pas attesté

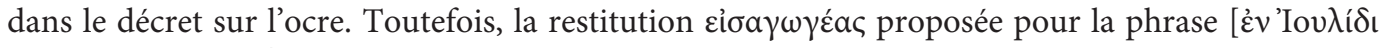

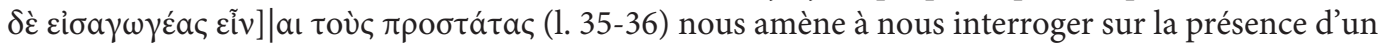
tribunal dans cette cité aussi. Mis à part ces détails, rien d'autre n'est connu sur leur composition et leur fonctionnement.

Quant à Délos, elle constitue un cas particulier, en raison de l'influence directe d'Athènes sur l'île au cours de la période classique, à l'occasion de la fondation de la Ligue de Délos et de

(73) Des fonctions judiciaires semblent également détenues par les prostatai à Calymna et Cos, dans le règlement des procès jugés par Cnide entre la cité de Calymna et les enfants de Diagoras au début du III ${ }^{\mathrm{e}}$ siècle (IK Knidos I, 221), et à Iasos, dans le règlement relatif à la prêtrise et au culte du Zeus Megistos (Iasos 2, 1. 7).

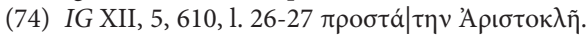

(75) IG XII, 5, 802, 1. 3-4 (III ${ }^{\mathrm{e}}$ siècle): décret en l'honneur de Nymphaios de Byzance; IG XII, 5, 824, B, 1. 34 (II ${ }^{\mathrm{e}}$ siècle): décret des Nésiotes en l'honneur du médecin Apollonios de Milet. Sur ces décrets, voir Étienne 1990, p. 95-97, 120-123.

(76) Voir Étienne 1990, p. 93 et n. 32, 34. Nous pensons avec lui que l'idée d'une magistrature d'exception, comme à Thasos (IG XII, 8, 262, 264), semble moins probable. Du reste, les prostatai sont chargés de fonctions similaires à celles évoquées dans les inscriptions de Ténos dans d'autres régions du monde grec (voir, e.g., Calymna, Cnide, Cos).

(77) Voir, e.g., Andros: IG XII, Suppl. 244 (IV siècle); Arkésinè d'Amorgos: IG XII, 7, 5 (IV siècle); Ios: IG XII, 5, 1002, 1004 (III ${ }^{\mathrm{e}}$ siècle); Ioulis de Kéos: IG XII, 5, 593, B, 1. 1-2 (v siècle); Karthaia de Kéos: IG XII, 5, 528, 538 et suppl. (Ive/ III $^{\mathrm{e}}$ siècle); Korèsia de Kéos: $I G \mathrm{II}^{2}, 1128$ (IV siècle); Naxos: SEG 33.676 (ca. 300); Paros: IG XII, 5, 110, 111 (IV ${ }^{\mathrm{e}}$ siècle); Sikinos: IG XII, 5, 24 (IV $/ \mathrm{III}^{\mathrm{e}}$ siècle).

(78) Sur les compétences judiciaires du Conseil athénien, voir RHodes 1972, p. 145-178. 
l'administration du sanctuaire d'Apollon ${ }^{79}$. On sait qu'à Athènes, au début de chaque année, six mille juges non professionnels étaient tirés au sort et siégeaient dans des tribunaux aux effectifs variables (la composition variait en fonction du type d'affaire), afin de juger la grande majorité des procès de la cité. Ces six mille Athéniens s'appelaient héliastes et formaient l'Héliée, le tribunal populaire d'Athènes. Le mot «Héliée» est attesté également dans quatre inscriptions de la Délos des $\mathrm{III}^{\mathrm{e}}$ et $\mathrm{II}^{\mathrm{e}}$ siècles $(2 \mathrm{a}-\mathrm{d})$. Bien que dans la quatrième inscription datant du $\mathrm{II}^{\mathrm{e}}$ siècle le terme puisse faire allusion à l'édifice qui accueille des réunions judiciaires, l'absence d'informations supplémentaires ne nous permet pas de tirer une conclusion ferme à propos de l'existence d'un lieu ou bâtiment qui portait le nom d' «Héliée». Le terme signifiait très probablement soit «tribunal populaire» (l'institution) soit, par synecdoque, «séance» de ce tribuna ${ }^{80}$. Même si les témoignages correspondants sont postérieurs à l'époque classique, l'existence d'une Héliée à Délos au III siècle est très intéressante, puisque Délos est à cette époque indépendante. Se pose alors la question suivante: une Héliée existait-elle déjà au $\mathrm{IV}^{\mathrm{e}}$ siècle, pendant le contrôle du sanctuaire d'Apollon par les Athéniens, ou les Déliens indépendants l'avaient-ils empruntée à Athènes? La question reste ouverte, puisqu'on manque d'informations sur les tribunaux de Délos au cours de la période classique. Dans un acte amphictyonique de Délos, datant du IV siècle, on trouve la référence à un

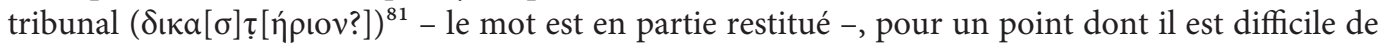
tirer des conclusions, et ce pour deux raisons. D'une part, on dispose d'inscriptions du même genre,

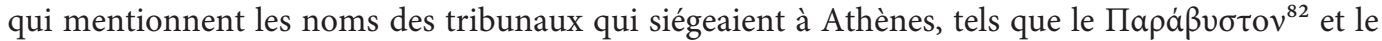

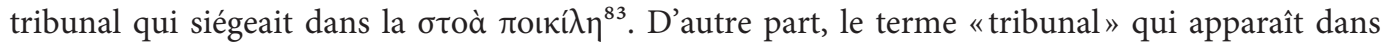
les inscriptions relatives à la procédure de l'adjudication des travaux qui ont eu lieu à Délos doit faire allusion à des tribunaux situés à Athènes ${ }^{84}$. Le seul cas pour lequel on est mieux renseigné et qui a apparemment été jugé à Délos en $376 / 5$ est un procès pour sacrilège ${ }^{85}$. Selon l'inscription, un groupe de Déliens a été accusé d'impiété et condamné à une amende et à l'exil perpétuel pour avoir traîné hors du sanctuaire d'Apollon et frappé les Amphictyons athéniens.

\section{Ekklèsia}

D’après l'inscription très fragmentaire trouvée à Théra (8), l'Assemblée de la cité se réservait

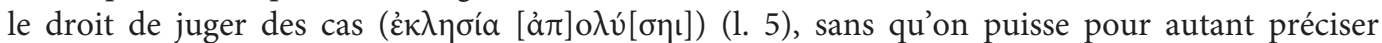
leur nature ${ }^{86}$. L'Assemblée de Siphnos disposait très probablement des mêmes compétences, sous réserve qu'on accepte la première restitution qui a été proposée pour le décret d'Athènes à propos de Siphnos (7a). Selon cette restitution, le peuple de Siphnos n'est pas autorisé à exécuter ou à poursuivre un Athénien, sans l'accord du peuple des Athéniens, pour éviter qu'un Athénien ne meure, sans jugement préalable (1.9-13). Au contraire, la restitution récente (7b) identifie les

(79) Sur un résumé de l’histoire de Délos classique, indépendante et athénienne, voir Guide de Délos, p. 35-43. Pour la période classique, voir surtout Chankowski 2008. Pour les relations tendues entre Athènes et Délos pendant le Iv ${ }^{\mathrm{e}}$ siècle, voir aussi CONSTANTAKOPOULOU 2016, p. 125-146.

(80) Voir sur ce point, MoRetTi 2001, p. 141-142.

(81) ID 97, 1. 27 (393-367).

(82) ID 104-22, b, 1. 12-13 (346/5).

(83) ID 104-26, C, 1. 5-7 (340).

(84) ID 104-4, a, A, 1. 28, B, 1.17 (360-350); ID 104-6, 1.24 (359/8); ID 104-19, A, 1.4 (434-315); ID 104-22, a, 1. 11-12, $18, \mathrm{~b}, 1.3(346 / 5)$.

(85) ID 98, B, a, 1. 24-30. Sur ce procès, voir CHANkowski 2008, p. 249-251 (suivie par BonNin 2015, p. 263), où elle discute l'hypothèse énoncée par DreHer 1995, selon lequel le procès aurait eu lieu à Athènes.

(86) Cf. Reger 2004, p. 783-784; Ager 2008, p. 154. L’Assemblée athénienne se réservait le droit jusqu'au milieu du $\mathrm{IV}^{\mathrm{e}}$ siècle de juger certaines affaires de nature politique (eisangélie). Voir à ce propos HaNSEN 1975. 
Siphniens comme défendeurs ${ }^{87}$, dont les cas qui entraînent les peines d'exil ou de mort doivent être jugés à Athènes.

\section{LE MATÉRIEL À FONCTION JUDICIAIRE: LES KLÈRÔTÈRIA}

Quatre fragments de machines pour le tirage au sort, appelées klèrôtèria, ont été trouvés sur l'île de $\operatorname{Délos}^{88}$. Sans que leur datation soit établie, celle de la période hellénistique étant la plus communément admise ${ }^{89}$, leur existence à Délos est révélatrice des relations de l'île avec Athènes. Si l'on voulait décrire brièvement cette machine, on dirait qu'il s'agissait d'un mécanisme utilisé à Athènes dans la procédure du tirage au sort à plusieurs niveaux, tels que la répartition des conseillers dans leurs sièges dans le Bouleutèrion, le tirage au sort des magistrats, la succession des prytanies au cours de l'année politique, le tirage au sort des juges et leur répartition dans les tribunaux. C'est surtout dans ce dernier domaine que le mécanisme de klèrôtèria a trouvé sa meilleure expression.

Il est très probable que dans le cas d'Athènes les klèrôtèria aient été introduits dans le domaine judiciaire aux environs de la création de la seconde Confédération maritime, parallèlement à l'introduction des tablettes judiciaires qui attestaient l'identité des juges (elles portaient leurs noms, parfois leurs patronymes, le nom de leur dème et une lettre correspondant à la section à laquelle ils appartenaient) et servaient à leur répartition dans les tribunaux ${ }^{90}$. Le système est décrit par Aristote de manière très précise dans les chapitres LXIII-LXV de la Constitution d'Athènes. Il fonctionnait sur la base des tribus et à chaque tribu correspondaient deux machines pour le tirage au sort des

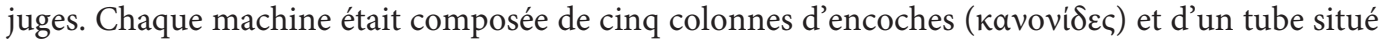
à gauche des stèles. Dans les encoches on glissait les tablettes des juges, et dans le tube on mettait

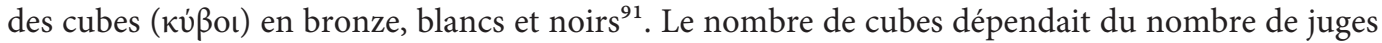
qui étaient nécessaires au jour du tirage au sort, puisqu'un cube comptait pour cinq tablettes, les cubes blancs correspondant à la désignation des juges, les noirs signifiant que les juges en question ne siégeraient pas ce jour-là. Ainsi, un jet de dés décidait si une série horizontale de cinq noms siégeait ou non au tribunal.

Les klèrôtèria trouvés à Délos ont été utilisés eux aussi, très probablement, pour le tirage au sort des héliastes. Ils se rapprochent de la description donnée par Aristote, puisqu'ils portent cinq colonnes de rainures et un tube placé à gauche des rainures. Ils présentent, en même temps, des similitudes avec deux fragments de klèrôtèria trouvés dans une église de Paroikia à Paros ${ }^{92}$. Ces derniers ont été fabriqués d'après le modèle athénien et portaient, comme ceux de Délos, un tube intégré dans la machine. On ne dispose cependant pas d'informations supplémentaires ni sur leur datation - ils doivent se situer entre le $\mathrm{IV}^{\mathrm{e}}$ et le $\mathrm{II}^{\mathrm{e}}$ siècle? - ni sur leur usage précis (bouleutique, judiciaire, etc.) ni, comme déjà évoqué, sur les tribunaux qui fonctionnaient à Paros. On n'est donc

(87) Voir Matthaiou 2010, p. 51.

(88) Ces quatre fragments sont publiés par MoRetTi 2001, p. 137-140.

(89) Voir Moretti 2001, p. 142.

(90) La monographie de Kroll 1972 reste l'étude principale sur les tablettes judiciaires. À celle-ci s'ajoute la découverte de nouvelles tablettes: Kroll 1984, p. 165; Boegehold et al. 1995, p. 64 (P19, P23); SEG 48.85; SEG 50.275; SEG 51.319; SEG 54.402, 403; SEG 55.326, 327; SEG 56.319, 320; GALANAKIS, SKALtSA 2012, p. 628-634.

(91) Sur la description de ce mécanisme, voir Dow 1939, p. 1-8, 23-25; Bishop 1970, p. 1-14; Boegehold et al. 1995, p. 33; Moretti 2001, p. 133. Un ouvrage, issu de la thèse de L. Rabatel, Klèrôtèria. Le tirage au sort dans le monde grec antique: machines, institutions et usages <http://theses.univ-lyon2.fr/documents/lyon2/2011/lopez_l/info >, est en cours de préparation, qui comprendra le dossier des klèrôtèria athéniens, ainsi que les quelques exemplaires découverts dans les Cyclades, à Délos et à Paros <http://www.iraa.mom.fr/recherche-activites/autres-recherches/publications-des-kleroteria $>$.

(92) Voir MüLler 1998, p. 167-172. 
pas en mesure de se fier aux klèrôtèria pariens conjointement avec l'inscription discutée auparavant sur le règlement relatif à la protection des arbres autour des sanctuaires de l'île (6), afin d'énoncer des hypothèses sur le système judiciaire à Paros.

\section{CONCLUSION}

En faisant le bilan des sources présentées, il convient de noter, tout d'abord, qu'en raison de l'absence fréquente d'informations sur d'autres régions du monde grec, hors d'Athènes, ou de l'état fragmentaire des sources disponibles, la comparaison avec Athènes est inévitable dans le cadre de la recherche de parallèles et de réponses sur le fonctionnement de ces cités. Sur la base de cette comparaison, il s'avère que les informations sur les Cyclades sont disparates et fragmentaires, de sorte que les lacunes importantes qu'elles présentent restreignent la portée de nos observations. Dans la mesure du possible, on peut trouver des similitudes entre le droit attique et les droits des cités voisines, ainsi qu'entre les droits des îles cycladiques elles-mêmes. Les différences entre eux ne manquent cependant pas et sont identifiées dans les procédures judiciaires et dans les pouvoirs des magistrats de ces cités, même dans les cas où ils portent des noms communs. En effet, ces différences prouvent qu'Athènes n'a pas nécessairement fourni un modèle adopté par les autres régions et qu'il n'existait pas de forme d'organisation commune à l'ensemble de ces îles, mettant en œuvre un droit commun. De surcroît, elles sont révélatrices des influences que les droits des cités pouvaient exercer sur leurs voisins compte tenu de la proximité géographique et des relations qui ont été établies au temps de la Ligue de Délos et de la seconde Confédération maritime. Même si, pendant le IV siècle et dans des cas particuliers, Athènes continue de faire transférer le jugement de certaines affaires de ses alliés devant ses tribunaux, l'existence d'aspects judiciaires communs est plutôt due à la coexistence continue des cités qu'à la pratique athénienne d'imposer ses procédures aux autres cités ${ }^{93}$. Du reste, les points communs qui sont repérés dans le dispositif judiciaire entre Athènes, ses alliés et des régions du monde grec de la même période qui n'appartiennent pas à la sphère athénienne manifestent l'existence d'un certain nombre de principes partagés par les communautés grecques, au-delà des seules Cyclades. Les témoignages fragmentaires dont on dispose pour la période classique augmentent de manière significative au cours de l'époque hellénistique, comme nous l'avons déjà vu pour le cas de Délos, les inscriptions fournissant toujours de nouvelles bases d'étude.

Alexandra BARTZOKA Paris-Sorbonne

(93) Cf. R \& O, p. 208-209. 


\section{Bibliographie}

ACCAME, S., 1941, La lega ateniese del secolo IV a.C., Rome.

Ager, S., 2008, «Rescuing Local History: Epigraphy and the Island of Thera», in G. Cooper (éd.), Epigraphy and the Greek Historian, Toronto, p. 150-176.

Arnaoutoglou, I., 1998, Ancient Greek Laws, Londres, New York.

Bishop, J. D., 1970, «The Cleroterium», JHS 90, p. 1-14.

Boegehold, A.L. et al., 1995, The lawcourts at Athens: sites, buildings, equipment, procedure, and testimonia (The Athenian Agora 28), Princeton N.J.

Bonnin, G., 2015, De Naxos à Amorgos. L'impérialisme athénien vu des Cyclades à l'époque classique, Bordeaux. Bonnin, G., LE Quéré, E., (éd.), 2014, Pouvoirs, îles et mer. Formes et modalités de l’hégémonie dans les Cyclades antiques (VII ${ }^{e}$ s. a.C.-III ${ }^{e}$ s. p.C.), Bordeaux.

Bresson, A., 2008, L'économie de la Grèce des cités. II. Les espaces de l'échange, Paris.

Brock, R., 2009, "Did the Athenian Empire Promote Democracy?», in J. Ma, N. Papazarkadas, R. Parker (éd.), Interpreting the Athenian Empire, Londres, p. 149-166.

Brun, P., 1989, «L'île de Kéos et ses cités au IV siècle av. J.-C.», ZPE 76, p. 121-138.

-, 2005, Impérialisme et démocratie à Athènes. Inscriptions de l'époque classique, Paris.

Bruneau, Ph., Ducat, J., 2005², Guide de Délos, Athènes.

Cargill, J.L., 1981, The Second Athenian League. Empire or Free Alliance?, Berkeley.

CARlier, P., 1995, Le IV siècle grec jusqu'à la mort d'Alexandre, Paris.

Carrara, A., 2014, "À la poursuite de l'ocre kéienne (IG, $\left.\mathrm{II}^{2}, 1128\right)$ : mesures économiques et formes de domination athénienne dans les Cyclades au IV s. a.C.», in BonNIn, LE QuÉré 2014, p. 295-316.

Cataldi, S., 1979, "Atene 'polis ekkletos' », ASNP III 9, p. 1-37.

Chandezon, Chr., 2003, L'élevage en Grèce (fin v'-fin I ${ }^{\text {er }}$ s. a. C.). L'apport des sources épigraphiques, Paris.

Chankowski, V., 2008, Athènes et Délos à l'époque classique. Recherches sur l'administration du sanctuaire d'Apollon délien, Athènes.

Constantakopoulou, Chr., 2007, The Dance of the Islands. Insularity, Networks, the Athenian Empire, and the Aegean World, Oxford.

—, 2016, "The Shaping of the Past: Local History and Fourth-century Delian Reactions to Athenian Imperialism», in A. Powell, K. Meidani (éd.), The Eyesore of Aigina: Anti-Athenian attitudes across the Greek, Hellenistic and Roman Worlds, Swansea, p. 125-146.

-, 2017, Aegean Interactions. Delos and its Networks in the Third Century, Oxford.

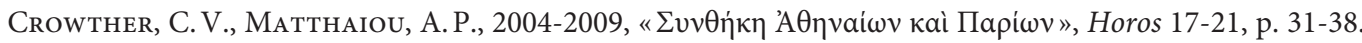

Dow, St., 1939, "Aristotle, the Kleroteria, and the Courts», HSPh 50, p. 1-34.

Drener, M., 1989, «Zu IG II ${ }^{2}$ 404, dem athenischen Volksbeschluss über die Eigenstaatlichkeit der keischen Poleis», Symposion 1985, Vienne, p. 263-281.

-, 1995, Hegemon und Symmachoi. Untersuchungen zum zweiten athenischen Seebund, Berlin, New York.

Étienne, R., 1990, Ténos II. Ténos et les Cyclades du milieu du IV siècle av. J.-C. au milieu du III siècle ap. J.-C., Athènes.

Finley, M. I., 1975, The Use and Abuse of History, Londres.

-, 1985, Studies in Land and Credit in Ancient Athens, 500-200 B.C. The Horos Inscriptions. With a New Introduction by Paul Millett, New Brunswick N.J.

FröHlich, P., 2004, Les cités grecques et le contrôle des magistrats (IV -I $^{\text {er }}$ siècle av. J.-C.), Genève.

Gagarin, M., 2005, «The Unity of Greek Law», in M. Gagarin, D. Cohen (éd.), The Cambridge Companion to Ancient Greek Law, New York, p. 29-40.

Galanakis, Y., Skaltsa, St., 2012, «Tomb robbers, art dealers, and a dikast's pinakion from an Athenian grave», Hesperia 81 , p. 619-653.

Game, J., 2008, Actes de vente dans le monde grec. Témoignages épigraphiques des ventes immobilières, Lyon. 
Gauthier, Ph., 1972, Symbola. Les étrangers et la justice dans les cités grecques, Nancy.

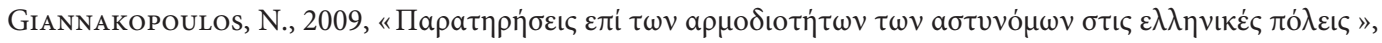
Egnatia 13, p. 9-21.

Hallof, Kl., Koerner, R., (éd.), 1993, Inschriftliche Gesetzestexte der frühen griechischen Polis, Köln, (=Inschriftliche).

Hamon, P., 2012, «Mander des juges dans la cité: notes sur l'organisation des missions judiciaires à l'époque hellénistique», Cahiers Glotz XXIII, p. 195-222.

Hansen, M. H., 1975, Eisangelia. The Sovereignty of the People's Court in Athens in the Fourth Century B.C. and the Impeachment of Generals and Politicians, Odense.

-, 1976, Apagoge, Endeixis and Ephegesis against Kakourgoi, Atimoi and Pheugontes. A Study in the Athenian Administration of Justice in the Fourth Century B.C., Odense.

Hansen, M.H., Nielsen, T.H., (éd.), 2004, An Inventory of Archaic and Classical Poleis, Oxford.

Harris, Ed. M., 2006, Democracy and the Rule of Law in Classical Athens. Essays on Law, Society and Politics, Cambridge.

—, 2008, Demosthenes, Speeches 20-22, Austin.

-, 2013a, "The Against Meidias (Dem. 21)», in M. Canevaro, The Documents in the Attic Orators. Laws and Decrees in the Public Speeches of the Demosthenic Corpus, Oxford, p. 209-236.

—, 2013b, "Finley's Studies in Land and Credit Sixty Years Later», Dike 16, p. 123-146.

Kroll, J.H., 1972, Athenian Bronze Allotment Plates, Cambridge Mass.

—, 1984, «More Athenian Bronze Allotment Plates», Studies presented to Sterling Dow (GRBS Monographs), p. $165-171$.

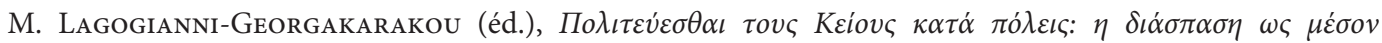

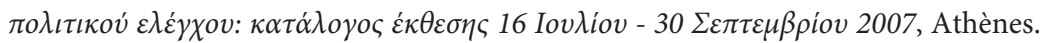

LARSEN, J. A. O., 1968, Greek Federal States. Their Institutions and History, Oxford.

LEwIS, D. M., 1997, Selected papers in Greek and Near Eastern history, Cambridge.

Low, P., 2007, Interstate Relations in Classical Greece, Cambridge.

MacDowell, D., 1978, The Law in Classical Athens, Londres.

—, 1991, «The Athenian procedure of phasis», Symposion 1990, Vienne, p. 187-198.

Magnetto, A., 2016, "Interstate Arbitration and Foreign Judges», in Ed. M. Harris, M. Canevaro (éd.), Oxford Handbook of Ancient Greek Law, Oxford, p. 1-43.

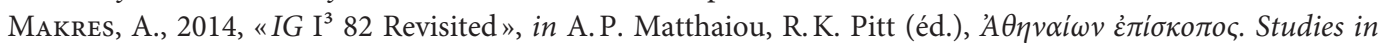
honour of H. B. Mattingly, Athènes, p. 185-202.

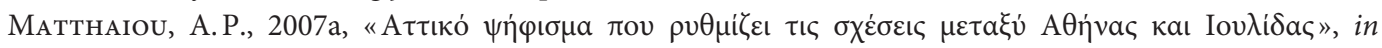
Lagogianni-Georgakarakou 2007, p. 38-41.

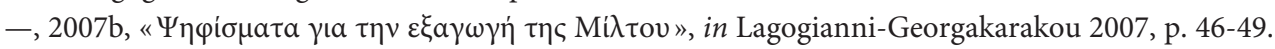

-, 2010, «A Treaty of Athens with Siphnos Revisited», in A. Tamis, C. J. Mackie, S. G. Byrne (éd.), $\Phi \iota \lambda \alpha \theta \dot{v} v \alpha \iota \varsigma$ - Philathenaios. Studies in honour of Michael J. Osborne, Athènes, p. 45-54.

MeIggs, R., 1972, The Athenian Empire, Oxford.

Migeotte, L., 2014, Les finances des cités grecques, Paris.

Moretti, J.-Ch., 2001, «Klèrôtèria trouvés à Délos», BCH 125, p. 133-143.

Müller, Kl., 1998, «Zwei Kleroterion-Fragmente auf Paros», AA, p. 167-172.

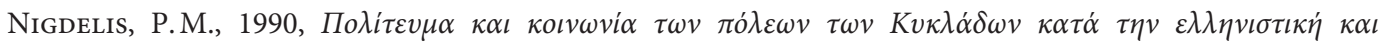

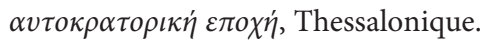

PÉbArthe, Chr., 2011, "L’empire athénien est-il toujours un empire comme les autres?», DHA Suppl. 5, p. $57-88$.

Pelloso, C., 2016, «Ephesis eis to dikasterion: Remarks and Speculations on the Legal Nature of the Solonian Reform», Symposion 2015, Vienne, p. 33-48. 
PiÉrart, M., 1971, «Les عủOvvol athéniens », AC 40, p. 526-573.

Reger, G., 2004, «The Aegean», in Hansen, Nielsen 2004, p. 732-793.

Rhodes, P. J., 1972, The Athenian Boule, Oxford.

- 2008, "After the three-bar sigma controversy: the history of Athenian imperialism reassessed», CQ 58, p. 501-506.

Rhodes, P. J., Lewis, D. M., 1997, The Decrees of the Greek States, Oxford.

Rhodes, P. J., Osborne, R., (éd.), 2003, Greek Historical Inscriptions, 404-323, Oxford, (=R \& O).

Robert, L., 2007, Choix d'écrits, Paris.

Rubinstein, L., 2003, «Volunteer Prosecutors in the Greek World», Dike 6, p. 87-113.

SCAFuro, A.C., 2015, «Decrees for Foreign Judges: Judging Conventions - or Epigraphical Habits?», Symposion 2013, Vienne, p. 365-395.

SCheibelreiter, Ph., 2013, Untersuchungen zur vertragsrechtlichen Struktur des delisch-attischen Seebundes, Vienne.

Sokolowski, F., 1962, Lois sacrées des cités grecques. Supplément, Paris, (=LSCG suppl.).

Sokolowski, F., 1969, Lois sacrées des cités grecques, Paris, (=LSCG).

Ste Croix de, G. E. M., 1961, «Notes on Jurisdiction in the Athenian Empire. I», CQ 11, p. 94-112.

VÉLISSAropoulos, J., 1980, Les nauclères grecs, Genève, Paris.

VIAL, Cl., 1985, Délos indépendante (314-167 av. J.-C.). Étude d'une communauté civique et de ses institutions (BCH Suppl. 10), Athènes.

Wallace, R. W., 2003, «Phainein in Athenian laws and legal procedures », Symposion 1999, Vienne, p. 167-181.

WolfF, H.J., 1972, «Die Bedeutung der Epigraphikfür die griechische Rechtsgeschichte», Vestigia 17, p. 131-144.

WoodheAD, A. G., 1957, «Greek Inscriptions », Hesperia 26, p. 221-236.

—, (éd.), 1997, The Athenian Agora. Volume XVI. Inscriptions. The Decrees, Princeton, (=Agora XVI). 
